\title{
Modeling water emission from low-mass protostellar envelopes ${ }^{\star}$
}

\author{
T. A. van Kempen ${ }^{1}$, S. D. Doty ${ }^{2}$, E. F. van Dishoeck ${ }^{1,3}$, M. R. Hogerheijde ${ }^{1}$, and J. K. Jørgensen ${ }^{4}$ \\ ${ }^{1}$ Leiden Observatory, Leiden University, PO Box 9513, 2300 RA Leiden, The Netherlands \\ e-mail: kempen@strw.leidenuniv.nl \\ 2 Department of Physics and Astronomy, Denison University, Olin 109 Granville, OH 43023, USA \\ 3 Max-Planck Institut für Extraterrestrische Physik (MPE), Giessenbachstr. 1, 85748 Garching, Germany \\ 4 Argelander-Institut für Astronomie, Universität Bonn, Auf dem Hügel 71, 53121 Bonn, Germany \\ Received 18 January 2008 / Accepted 3 April 2008
}

ABSTRACT

\begin{abstract}
Context. Within low-mass star formation, water vapor plays a key role in the chemistry and energy balance of the circumstellar material. The Herschel Space Observatory will open up the possibility to observe water lines originating from a wide range of excitation energies.

Aims. Our aim is to simulate the emission of rotational water lines from envelopes characteristic of embedded low-mass protostars. A large number of parameters that influence the water line emission are explored: luminosity, density, density slope, and water abundances.

Methods. Both dust and water emission are modeled using full radiative transfer in spherical symmetry. The temperature profile is calculated for a given density profile. The $\mathrm{H}_{2} \mathrm{O}$ level populations and emission profiles are in turn computed with a non-LTE line code. The results are analyzed to determine the diagnostic value of different lines, and are compared with existing observations.

Results. Lines can be categorized in: (i) optically thick lines, including ground-state lines, mostly sensitive to the cold outer part; (ii) highly excited $\left(E_{\mathrm{u}}>200-250 \mathrm{~K}\right)$ optically thin lines sensitive to the abundance in the hot inner part; and (iii) lines which vary from optically thick to thin depending on the abundances. Dust influences the emission of water significantly by becoming optically thick at the higher frequencies, and by pumping optically thin lines.

Conclusions. A good physical model of a source, including the correct treatment of dust, is a prerequisite for inferring the water abundance structure and possible jumps at the evaporation temperature from observations. The inner warm $(T>100 \mathrm{~K})$ envelope can be probed by highly excited lines, while a combination of excited and spectrally resolved ground state lines probes the outer envelope. Observations of $\mathrm{H}_{2}^{18} \mathrm{O}$ lines, although weak, provide even stronger constraints on abundances.
\end{abstract}

Key words. stars: pre-main sequence - circumstellar matter - stars: formation - ISM: molecules - submillimeter

\section{Introduction}

Low-mass $\left(M<3 M_{\odot}\right)$ young stellar objects (YSOs) form through gravitational collapse of cloud cores. Most of their mass is accreted over periods of less than $10^{6}$ years (André et al. 2000; Myers et al. 2000). These early stages of low-mass star formation, the so-called Class 0 and Class I stages, are characterized by the presence of centrally condensed envelopes that veil the central protostars and disks (Lada 1987; André et al. 1993). The envelopes also contain most of the mass $\left(M \sim 0.1-1 M_{\odot}\right)$ of the total system until the emergence of the central star and disk (Adams et al. 1987; Shirley et al. 2000; Jørgensen et al. 2002). Water plays a pivotal role in these early evolutionary stages. First, the high dipole moment of $\mathrm{H}_{2} \mathrm{O}$ makes its rotational lines excellent coolants of warm gas in the inner region of protostellar envelopes, allowing material to continue collapsing at higher temperatures (Goldsmith \& Langer 1978; Neufeld \& Kaufman 1993; Ceccarelli et al. 1996). Second, water is one of the dominant reservoirs of the non-refractory oxygen budget in dense clouds (van Dishoeck et al. 1993). The partitioning of gaseous oxygen between $\mathrm{H}_{2} \mathrm{O}, \mathrm{OH}$ and $\mathrm{O}$, as well as the ratio between $\mathrm{H}_{2} \mathrm{O}$ gas and ice, affect the chemistry of all other oxygen-containing molecules including the complex organics

^ Figures 11-14 and Tables 9-12 are only available in electronic form at http://www . aanda.org
(Charnley et al. 1992). The evaporation point of $\mathrm{H}_{2} \mathrm{O}$ ice in interstellar space is around $100 \mathrm{~K}$ (Fraser et al. 2001), so only the small warm inner regions of protostellar envelopes are likely to contain fractional abundances of water vapor as high as $10^{-4}$ with respect to $\mathrm{H}_{2}$, perhaps even exceeding that of $\mathrm{CO}$ (e.g., Ceccarelli et al. 1996; van Dishoeck \& Blake 1998; Boonman et al. 2003). In colder regions, water is the main ingredient of icy grain mantles as shown by infrared spectroscopy (e.g., Whittet et al. 1983; Smith et al. 1989; Boogert et al. 2002; Pontoppidan et al. 2004), with gas-phase water abundances that are orders of magnitudes lower. This abundance jump makes water an excellent tracer of warm, dense gas and a unique probe into the inner region close to the forming star.

Observations of interstellar gas-phase water from the ground are limited by the large amounts of water vapor in the Earth's atmosphere. Water maser lines at radio wavelengths longward of $3 \mathrm{~mm}(100 \mathrm{GHz})$, such as at $22 \mathrm{GHz}$, are readily seen, even from low-mass embedded YSOs (e.g., Furuya et al. 2003; Park \& Choi 2007), but are constrained to small hot spots and are often variable in nature (Felli et al. 2007). They provide little information about the general physical and chemical evolution during star formation (Elitzur et al. 1989). One of the few exceptions of a non-masing water line observable from the ground is the $183 \mathrm{GHz} J=3_{13}-2_{02}$ transition, which can be used to trace widespread water emission under exceptional conditions 
(e.g., Cernicharo et al. 1990, 1996; Doty 2000). Observations of deuterated water can also be done from the ground, in particular through the HDO $1_{01}-0_{00} 464 \mathrm{GHz}, 3_{12}-2_{21} 225 \mathrm{GHz}$, and $2_{11}-2_{12} 241 \mathrm{GHz}$ lines (e.g., Turner et al. 1975; Jacq et al. 1990; Helmich et al. 1996; Stark et al. 2004; Parise et al. 2005) and the $\mathrm{D}_{2} \mathrm{O} 1_{10}-1_{01} 316 \mathrm{GHz}$ line (Butner et al. 2007), but the large uncertainties in the $\mathrm{D} / \mathrm{H}$ ratio owing to fractionation make such observations a poor tracer of water itself. The $\mathrm{H}_{2}^{18} \mathrm{O}$ isotopologue can be detected as well, but only through its high excitation temperature $\left(E_{\text {up }}=193 \mathrm{~K}\right) 3_{13}-2_{20}$ transition at $203 \mathrm{GHz}$ (e.g., Jacq et al. 1988; Gensheimer et al. 1996; van der Tak et al. 2006).

To observe water emission lines systematically for a wide range of energy states, one has to turn to space-based observatories. Gaseous water has been detected in a number of starforming regions using the Infrared Space Observatory (ISO), the Sub-millimeter Wave Astronomy Satellite (SWAS), and ODIN (e.g., Helmich et al. 1996; Harwit et al. 1998; Nisini et al. 1999; Ceccarelli et al. 1999; Snell et al. 2000; Bergin \& Snell 2002; Maret et al. 2002; Nisini et al. 2002; Boonman et al. 2003; Bergin \& Melnick 2005; Ristorcelli et al. 2005; Cernicharo \& Crovisier 2005). However, due to large beams, low spectral resolution, or a combination of both, such observations were unable to accurately resolve the water abundance profile and trace the origin of the water emission, especially for low-mass YSOs. Two instruments onboard the Herschel Space Observatory ${ }^{1}$ can be used for future observations of rotational water lines at unprecedented sensitivity, resolution and spectral coverage (Ceccarelli et al. 2001). The Heterodyne Instrument for the Far Infrared (HIFI) (de Graauw \& Helmich 2001) and Photodetector Array Camera and Spectrometer (PACS) (Poglitsch et al. 2001) provide high spectral (up to $125 \mathrm{kHz}$ ) and spatial $\left(20^{\prime \prime}\right.$ at $1 \mathrm{THz}$ ) resolution, which will allow a thorough analysis of water emission through a much wider range of observable transitions. In addition, the high sensitivity of the instrument will allow observations of various lines at low abundance, such as the optically thin isotopologues of water, in particular $\mathrm{H}_{2}^{18} \mathrm{O}$. However, to infer abundances from such observations, detailed radiative transfer calculations are needed.

Several modeling efforts have been carried out to analyze the observed water lines, based either on models of shocks at high and low velocities (e.g., Kaufman \& Neufeld 1996; Giannini et al. 2001; Nisini et al. 2002) or on thermal emission from the envelope itself (e.g., Maret et al. 2002; Boonman et al. 2003). As pointed out by van der Tak et al. (2006), the high water abundance combined with its high dipole moment and its susceptibility to far-infrared emission from dust results in high optical depths make this molecule particularly difficult to model. As a result, no systematic parameter studies have been carried out yet We present here a parameter study of the water emission from low-mass protostellar envelopes using a full radiative transfer method including the effects of dust that is applied to realistic physical models of low-mass YSOs. Specific predictions for Herschel are made by convolving the computed emission with the Herschel observing beams. Poelman \& van der Tak (2007) present similar models for high-mass protostars.

This paper is organized as follows. Section 2 presents the physical and molecular parameters necessary to accurately model water. Section 3 takes an in-depth look at a single Class 0 source, L 483, and explores the effects of possible water abundance profiles. Section 4 presents the results from the range of parameters that influence water emission. Section 5 investigates the influence of dust on water line fluxes and profiles, which

${ }^{1}$ See http://herschel.esac.esa.int/
Table 1. Parameters for low-mass protostellar envelopes.

\begin{tabular}{ll}
\hline \hline \multicolumn{2}{c}{ Variables } \\
\hline$n_{\mathrm{H}_{2}}(1000 \mathrm{AU})\left(10^{6} \mathrm{~cm}^{-3}\right)$ & $0.4,1,5$ \\
$p$ & $1.5,2.0$ \\
Luminosity $\left(L_{\odot}\right)$ & $2,7,25$ \\
$X_{0}$ (water) & $10^{-4}, 10^{-6}$ \\
$X_{\mathrm{d}}$ (water) & $10^{-6}, 10^{-7}, 10^{-8}$ \\
\hline \multicolumn{2}{c}{$\quad$ Fixed parameters } \\
Distance & $150 \mathrm{pc}$ \\
Stellar temperature & $5000 \mathrm{~K}$ \\
Gas temperature & $=T_{\text {dust }}$ \\
$R_{\text {in }}$ (inner radius) & $250 \mathrm{~K}$ \\
$R_{\text {out }}$ (outer radius) & $200 R_{\text {in }}$ \\
$r_{0}$ & $1000 \mathrm{AU}$ \\
Water freeze-out & $100 \mathrm{~K}$ \\
$o: p$ ratio $\mathrm{H}_{2} \mathrm{O}^{a}$ & $3: 1$ \\
$o: p$ ratio $\mathrm{H}_{2}$ & $1: 1$ \\
Dust properties & $\mathrm{OH} 5^{b}$ \\
Velocity dispersion $\Delta V$ & $1.67 \mathrm{~km} \mathrm{~s}^{-1}$ \\
\hline
\end{tabular}

${ }^{a}$ For the L483 models, ortho-, and para-water were modeled independently. See Sect. $3 ;^{b}$ see Ossenkopf \& Henning (1994) Col. 5.

is often ignored. Section 6 gives an analysis of a selected sample of observed water lines from ISO, SWAS, and ODIN to test the models and to infer implications for observations with future instruments such as HIFI and PACS. Final conclusions and recommendations can be found in Sect. 7.

\section{Model}

The modeling has been carried out in two stages. First, the dust radiative transfer is calculated for a given power-law density profile (Sect. 2.1). This produces dust temperature profiles for given density structures. Subsequently, water level populations and line emission profiles are modeled using the line radiative transfer code RATRAN (Sect. 2.2) (Hogerheijde \& van der Tak 2000), with the assumption that the gas temperature is equal to that of the dust. For an overview of the steps, see van Dishoeck (2003) (Fig. 2). Our method refers to the "empirical method" presented there. See Table 1 for an overview of the parameters.

\subsection{Physical structure of the envelope model}

\subsubsection{Dust radiative transfer}

Our protostellar envelope models are taken to be spherically symmetric, with the protostar located at the center. It has been shown that this assumption is valid for analyzing both the continuum and line emission down to scales of a few hundred AU (e.g., Shirley et al. 2000; Jørgensen et al. 2002; Schöier et al. 2002; Jørgensen et al. 2005). The density $n$ at radius $r$ within the protostellar envelope is described by

$n(r)=n_{0}\left(r / r_{0}\right)^{-p}$

with the power-law exponent $p$ and density $n_{0}$ at a reference radius $r_{0}$ as parameters. Within the context of protostellar collapse theories, both parameters depend on the age of the system and on the original core conditions, such as its initial mass.

Both the gas and dust temperatures inside the envelope are also often described by a power-law profile. However, this approximation fails at small radii where the dust becomes optically thick (e.g., Doty \& Leung 1994; Jørgensen et al. 2002; 
Table 2. The masses and inner radii of the model envelopes.

\begin{tabular}{ccccc}
\hline \hline$L_{\text {bol }}$ & $\begin{array}{c}n_{\mathrm{H}^{2}}\left(r_{0}\right) \\
L_{\odot}\end{array}$ & $\begin{array}{c}M^{6} \mathrm{~cm}^{-3} \\
M_{\odot}\end{array}$ & $\begin{array}{c}R_{\text {in }} \\
\mathrm{AU}\end{array}$ & $\begin{array}{c}R_{100 \mathrm{~K}} \\
\mathrm{AU}\end{array}$ \\
\hline & & $p=1.5$ & & \\
2 & 0.4 & 0.06 & 8.22 & 23 \\
2 & 1 & 0.15 & 8.22 & 23 \\
2 & 5 & 0.76 & 8.22 & 23 \\
7 & 0.4 & 0.15 & 15.3 & 42 \\
7 & 1 & 0.39 & 15.3 & 42 \\
7 & 5 & 1.93 & 15.3 & 42 \\
25 & 0.4 & 0.40 & 29 & 80 \\
25 & 1 & 1.01 & 29 & 80 \\
25 & 5 & 5.04 & 29 & 80 \\
\hline & & $p=2$ & & \\
2 & 0.4 & 0.07 & 8.22 & 21 \\
2 & 1 & 0.18 & 8.22 & 21 \\
2 & 5 & 0.90 & 8.22 & 21 \\
7 & 0.4 & 0.13 & 15.3 & 38 \\
7 & 1 & 0.33 & 15.3 & 38 \\
7 & 5 & 1.65 & 15.3 & 38 \\
25 & 0.4 & 0.24 & 29 & 72 \\
25 & 1 & 0.63 & 29 & 72 \\
25 & 5 & 3.15 & 29 & 72 \\
\hline
\end{tabular}

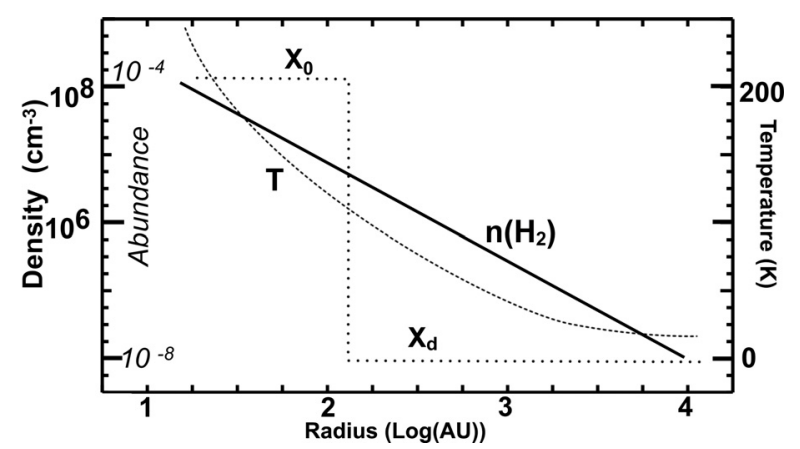

Fig. 1. Example of the physical structure of a model envelope with the density (solid), temperature (dashed), and abundance (dotted) displayed versus radius. The adopted parameters are $n_{0}\left(\mathrm{H}_{2}\right)=10^{6} \mathrm{~cm}^{-3}, p=1.5$, and $L=2 L_{\odot}$.

Young \& Evans 2005). The actual temperature structure depends on a combination of the density structure and the central source luminosity, and a dust radiative transfer calculation is needed to determine it. The radiation is produced by the energy released through accretion of material onto the protostar.

The continuum radiative transfer through the envelope was calculated using the spherically symmetric dust radiative transfer code DUSTY (Ivezić \& Elitzur 1997). DUSTY is a scalefree 1D code that uses the ratio $Y$ of the outer over inner radius, $R_{\text {out }} / R_{\text {in }}$, the dust opacity $\tau$ at $100 \mu \mathrm{m}$, the temperature at $R_{\text {in }}$, and the power-law index of the density profile $p$ as its variables. Results are then scaled by using the luminosity and distance of specific sources. The inner edge of our envelope $R_{\text {in }}$ was set at the radius where the temperature of the envelope reaches $250 \mathrm{~K}$. Material closer to the star is assumed to be located in a disk, which is not taken into account. In addition, we assume that the disk has no influence on the temperature and density distribution of the envelope. The exact inner radius in AU depends on the luminosity, which differs for each model, and $R_{\text {in }}$ is typically a few tens of AU, much smaller than the Herschel beams (see Table 2). In all our models, $Y$ is taken to be 200 . Values of $R_{\text {out }}$ are a few thousand to ten thousand $\mathrm{AU}$, greater than the Herschel beam $\left(20^{\prime \prime}\right.$ at $\left.1113 \mathrm{GHz}\right)$ at all but the lowest frequencies, but generally less than the values given in Jørgensen et al. (2002). The adopted dust opacity's are the often-used OH tables, Col. 5 of Ossenkopf \& Henning (1994) throughout. Figure 1 shows a typical temperature and density profile.

\subsubsection{Gas radiative transfer}

The gas temperature is taken to be equal to the dust temperature for the entire spatial extent of our model, and the gas:dust mass ratio is taken to be 100:1. Warm dense gas can efficiently cool through the emission of excited molecular and atomic lines such as $\mathrm{CO}, \mathrm{H}_{2} \mathrm{O}, \mathrm{OH}$, and [O I] (Giannini et al. 2001). Water can also heat the gas through far-infrared pumping followed by collisional de-excitation (e.g., Ceccarelli et al. 1996). As a result, dust and gas temperatures can differ slightly, especially in the 100-200 K range (e.g., Ceccarelli et al. 2000; Maret et al. 2002); however, at densities of $10^{7} \mathrm{~cm}^{-3}$ or higher, the gas and dust in the warm inner region couple efficiently. At low densities $\left(<10^{5} \mathrm{~cm}^{-3}\right)$ and temperatures $(<50 \mathrm{~K})$, any differences between gas and dust temperature depend on the source parameters (e.g., luminosity, total envelope mass) and the external interstellar radiation field (ISRF). Doty \& Neufeld (1997) show in their Fig. 12 that these differences are generally small, about $10 \mathrm{~K}$ or less for low-mass YSOs, and typically only exist at radii greater than 2000 AU. Boonman et al. (2003) show that for high-mass YSOs, the water line emission for a full radiative transfer calculation of the gas temperature differs negligible from that calculated using the $T_{\text {dust }}=T_{\text {gas }}$ assumption.

Our choice of $R_{\text {out }}=200 R_{\text {in }}$ assumes that water vapor at larger radii does not contribute to the total water line emission due to the low densities $\left(<10^{5} \mathrm{~cm}^{-3}\right)$ and temperatures $(<15 \mathrm{~K})$ in these regions. This assumption mostly affects the transitions leading to the ground states. Any such material is able to absorb water emission for optically thick lines, and lead to weak emission lines in observations taken with a beam that is much larger than our adopted source sizes. However, such extended envelope material is almost indistinguishable from any larger scale cloud complexes not directly related to the source. Such contributions from large-scale clouds need to be taken into account on a source-by-source basis, but are beyond the scope of this work. The protostellar envelopes are assumed to be shielded from strong external radiation fields by surrounding molecular cloud material. If directly exposed to outside radiation, the temperature in the outer envelopes is affected by the ISRF, but material is not heated by more than $40 \mathrm{~K}$ for the ISRF found within the Orion starforming region (Jørgensen et al. 2006). Low-mass starforming regions are expected to have significantly lower ISRF. External radiation is not considered for these models.

\subsubsection{Grid parameters}

To limit the model complexity, the wide range of parameters was reduced to four variables (see Table 1). The density at $1000 \mathrm{AU}, n_{0}\left(\mathrm{H}_{2}\right)$ and the exponent of the density power law $p$ are two of these. Jørgensen et al. (2002) observed many lowmass YSOs and inferred densities at 1000 AU between $1 \times 10^{5}$ to $5 \times 10^{6} \mathrm{~cm}^{-3}$. Accordingly, three values, $0.4 \times 10^{6}, 1 \times 10^{6}$, and $5 \times 10^{6} \mathrm{~cm}^{-3}$ were chosen to sample this range, and $p$ was chosen to be either 1.5 or 2.0. The stellar variables are reduced to a single variable, the luminosity. Models using luminosities of 2, 7, and $25 L_{\odot}$ are explored to represent the wide range of luminosities observed in low-mass protostars. Although the 

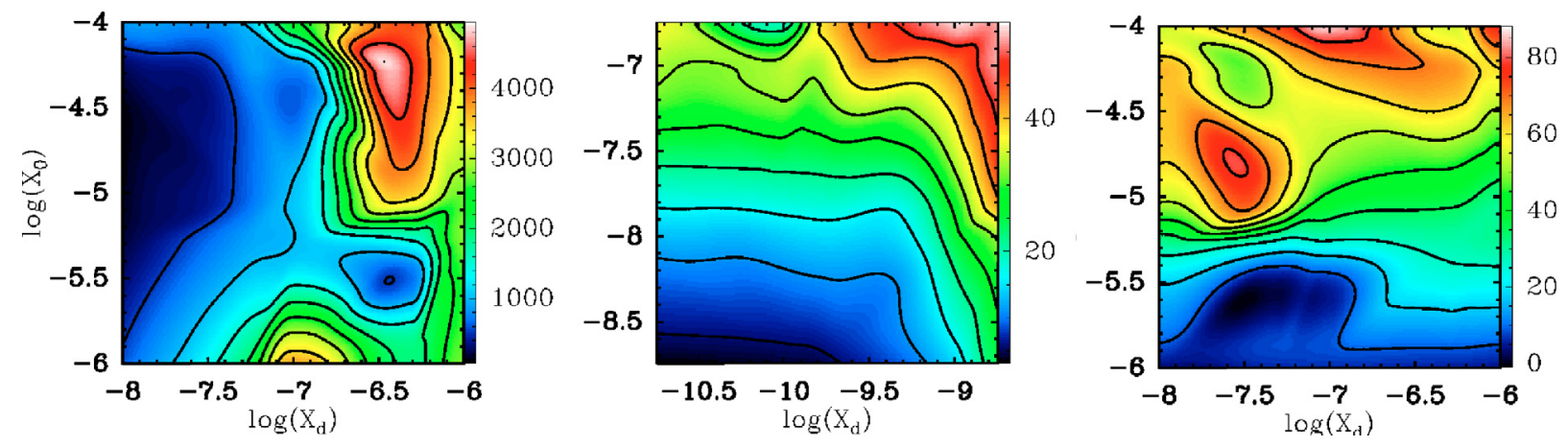

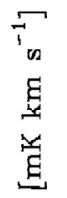

Fig. 2. Contour plots of the integrated water line intensity for the $\mathrm{L} 483$ models, plotted as functions of $X_{\mathrm{d}}$ and $X_{0}$. Left: the o- $\mathrm{H}_{2} \mathrm{O} 1_{10}-1_{01}$ line $(557 \mathrm{GHz})$, showing a strong dependence on $X_{\mathrm{d}}$; middle: the $\mathrm{p}-\mathrm{H}_{2}^{18} \mathrm{O} 2_{02}-1_{11}$ line $(995 \mathrm{GHz})$, showing a clear cut between the dependency on $X_{\mathrm{d}}$ or $X_{0}$, depending on the abundance profile; right: the $\mathrm{p}-\mathrm{H}_{2} \mathrm{O} 5_{24}-4_{31}$ line $(970 \mathrm{GHz})$, showing a strong dependence on $X_{0}$.

surface temperature of the protostar cannot be derived directly, it is assumed to be around $5000 \mathrm{~K}$ for low-mass young stars, regardless of the luminosity or age. Test models show that, due to the reprocessing of stellar photons into IR/thermal photons very close to the star, the stellar effective temperature has little influence on the modeling. The final variable is the gas-phase water abundance, to be discussed below. Table 1 summarizes all the adopted parameters. The resulting masses and inner and outer radii for each envelope model can be found in Table 2 .

\section{2. $\mathrm{H}_{2} \mathrm{O}$ line modelling}

Because of the gradient in temperature, the water abundance $X$ with respect to $\mathrm{H}_{2}$ is expected to vary within YSO envelopes. Previous studies of low- and high-mass YSOs used a variety of $\mathrm{H}_{2} \mathrm{O}$ observations with ISO-LWS, ISO-SWS, and/or SWAS to constrain the water abundance profile in protostellar envelopes to have a "jump" in abundance at a characteristic temperature around 100 K (e.g., Maret et al. 2002; Boonman et al. 2003; van der Tak et al. 2006). Such "jumps" have also been confirmed by ground-based observations of other molecules such as $\mathrm{CH}_{3} \mathrm{OH}$ and $\mathrm{H}_{2} \mathrm{CO}$ (e.g., van der Tak et al. 2000; Ceccarelli et al. 2000; Schöier et al. 2002; Jørgensen 2004; Maret et al. 2004). The abundance of water in the inner region $X_{0}$ is high for two reasons. First, most water exists in the gas phase above $100 \mathrm{~K}$, the temperature at which water ice evaporates from the grains. Second, gas-phase reactions of atomic oxygen with molecular hydrogen drive all oxygen into water at high temperatures $(T>230 \mathrm{~K})$ (Charnley et al. 2001). However, in regions where the temperature drops below $100 \mathrm{~K}$, water is mostly frozen out onto the grains, and only trace amounts of gaseous water are present with an abundance $X_{\mathrm{d}}$.

Accordingly, "jump" abundances are assumed for all models with $X_{0}$, the abundance of the warm inner region, taken to be $10^{-4}$ or $10^{-6}$, and $X_{\mathrm{d}}$, the abundance in the cold outer region, taken to be $10^{-6}, 10^{-7}$, or $10^{-8}$. These values cover the range of inferred abundances from observations to date for embedded protostars. Although beam-averaged $\mathrm{H}_{2} \mathrm{O}$ abundances below $10^{-8}$ have been observed for B 68 and Oph-D (Bergin $\&$ Snell 2002), such clouds are starless. At the extremely low temperatures of $<15 \mathrm{~K}$, virtually all heavy elements are frozen out. Abundances as low as $10^{-11}$ were tested by Boonman et al. (2003), but models with outer abundances of $10^{-8}$ were preferred in fitting the combined SWAS and ISO-LWS data. One combination of abundances is a constant abundance at $10^{-6}$, included to investigate the differences between "jump" and constant abundance profiles. A finer grid of abundances within this range is explored for the L 483 model. Figure 1 includes the assumed abundance structure. Our models do not consider a so-called "drop" abundance profile (see Schöier et al. 2004), in which the abundance in the outermost part is higher due to longer timescales for freeze-out at lower densities and due to photodesorption of ices (Bergin \& Melnick 2005). Such an abundance profile would result in more self-absorption in the ground-state lines, equivalent to absorption caused by the cold fore-ground material not associated with the protostellar system.

The effects of micro-turbulence within the envelope are represented by a velocity dispersion $F W H M, \Delta V=1.67 \mathrm{~km} \mathrm{~s}^{-1}(b=$ $1 \mathrm{~km} \mathrm{~s}^{-1}$, with $b$ the Doppler parameter), consistent with the typical line widths observed for optically thin rotational emission in low-mass YSO envelopes (Jørgensen et al. 2002, 2004, 2005). For a more thorough discussion of the effects of the choice of this value, see Sect. 5.2. An infall velocity profile was included, based on the velocity profiles for collapsing clouds as found by Shu (1977). This velocity profile was scaled to a velocity of $4 \mathrm{~km} \mathrm{~s}^{-1}$ at the inner radius $R_{\mathrm{in}}$. At radii of a few hundred $\mathrm{AU}$, the micro-turbulent velocity dominates over the infall velocity, which is the scale probed mostly by single-dish observations (e.g., Jørgensen et al. 2004). $\mathrm{H}_{2}$ is assumed to be in a $1: 1$ ortho to para ratio. Water itself is in $3: 1$ ortho to para ratio. Collisional rate coefficients for both ortho- and para- $\mathrm{H}_{2} \mathrm{O}$ with ortho- and para- $\mathrm{H}_{2}$ were obtained from the LAMDA database (Schöier et al. 2005) based on calculations by Green et al. (1993) for temperatures in the range from 20 to $2000 \mathrm{~K}$. New rate coefficients have recently been published (Phillips et al. 1996; Dubernet \& Grosjean 2002; Grosjean et al. 2003; Faure et al. $2007)^{2}$. For a general discussion of uncertainties in molecular data, see Schöier et al. (2005).

The excitation and radiative transfer of the rotational water lines were calculated using the spherically symmetric version of the RATRAN code (Hogerheijde \& van der Tak 2000). First, level populations were computed for all $\mathrm{H}_{2} \mathrm{O}$ levels with energies up to $2000 \mathrm{~K}$ for the model envelope using full Monte Carlo radiative transfer. Individual iterations use a convergence criterion

\footnotetext{
2 The rate coefficients from Faure et al. (2007) only became available once most of the models had been run, but those new calculations often do not include a wide enough range of temperatures or the higher excited energy levels, needed for the analysis of YSOs. Test models using different published rate coefficients show that absolute line fluxes may differ up to $30 \%$ (Poelman \& van der Tak 2007). Even greater differences are found at low temperatures $(T<20 \mathrm{~K})$ and densities $\left(n\left(\mathrm{H}_{2}\right)<10^{4} \mathrm{~cm}^{-3}\right)$ for the ground-state line intensities and profiles (e.g. Poelman \& Spaans 2005; Dubernet et al. 2006). However, the general trends found in this paper remain valid for different rate coefficients.
} 
Table 3. Key water lines observable with Herschel HIFI and PACS.

\begin{tabular}{ccccc}
\hline \hline Transition & $\begin{array}{c}\text { Freq. } \\
(\mathrm{GHz})\end{array}$ & $\begin{array}{c}\text { Wavelength } \\
(\mu \mathrm{m})\end{array}$ & $\begin{array}{c}E_{\text {up }} \\
(\mathrm{K})\end{array}$ & $\begin{array}{c}\text { Beam }^{a} \\
\left.{ }^{\prime \prime}\right)\end{array}$ \\
\hline \multicolumn{5}{c}{ HIFI } \\
Ortho-H $\mathrm{O}$ transition \\
$1_{10}-1_{01}$ & 556.93607 & 538.66147 & 61.0 & 39 \\
$2_{12}-1_{01}$ & 1669.90496 & 179.65094 & 114.4 & 13 \\
$2_{21}-2_{12}$ & 1661.00802 & 180.61322 & 194.1 & 13 \\
$3_{12}-2_{21}$ & 1153.12682 & 260.16219 & 249.4 & 19 \\
$3_{12}-3_{03}$ & 1097.36505 & 273.38213 & 249.4 & 21 \\
$3_{21}-3_{12}$ & 1162.91187 & 257.97312 & 305.3 & 19 \\
\hline \multicolumn{5}{c}{ Para- ${ }_{2} \mathrm{O}$ transition } \\
$1_{11}-0_{00}$ & 1113.34306 & 269.45872 & 53.4 & 21 \\
$2_{02}-1_{11}$ & 987.92670 & 303.66625 & 100.8 & 21 \\
$2_{11}-2_{02}$ & 752.03323 & 398.91854 & 136.9 & 30 \\
$2_{20}-2_{11}$ & 1228.78902 & 244.14281 & 195.9 & 19 \\
$3_{31}-4_{04}$ & 1893.68681 & 158.42113 & 410.4 & 13 \\
$4_{22}-3_{31}$ & 916.17164 & 327.44956 & 454.3 & 25 \\
$4_{22}-4_{13}$ & 1207.63890 & 248.41863 & 454.3 & 19 \\
$5_{24}-4_{31}$ & 970.31524 & 309.17787 & 598.8 & 21 \\
\hline \multicolumn{5}{c}{ PACS } \\
$2_{12}-1_{01}$ & 1669.90496 & 179.65094 & 114.4 & 13 \\
$2_{21}-1_{10}$ & 2773.97691 & 108.14798 & 194.1 & 8 \\
$2_{21}-2_{12}$ & 1661.00802 & 180.61322 & 194.1 & 13 \\
$3_{03}-2_{12}$ & 1716.76979 & 174.74678 & 196.8 & 12 \\
$4_{14}-3_{03}$ & 2640.47434 & 113.61595 & 323.5 & 8 \\
\hline \multicolumn{5}{c}{ Para- $\mathrm{H}_{2} \mathrm{O}$ transition } \\
$2_{20}-1_{11}$ & 2968.74895 & 101.05267 & 195.9 & 7 \\
$3_{13}-2_{02}$ & 2164.13230 & 138.62371 & 204.7 & 10 \\
$4_{04}-3_{13}$ & 2391.57290 & 125.44046 & 319.5 & 9 \\
\hline \multicolumn{5}{c}{ Ortho- ${ }_{2} \mathrm{O}$ transition } \\
\hline
\end{tabular}

${ }^{a}$ Taken from http://herschel. esac.esa.int/home.shtml

of $10^{-6}$ on the relative error of the fractional population for the solution of the statistical equilibrium. Iterations continue with increasing amounts of photons, until the greatest relative difference between fractional populations over all cells and all levels of three subsequent iterations is less than $1 / 6$. In practice this means that the vast majority of cells and levels are converged to much smaller relative errors. Far-infrared radiation from the dust and $\mathrm{CMB}$ are included in the $\mathrm{H}_{2} \mathrm{O}$ excitation and line formation. This inclusion makes it possible to investigate infrared pumping, self-absorption of a line, and absorption against the dust continuum. Even though many levels are very sparsely populated, tests show that it is necessary to include the higher levels to obtain an accurate population distribution throughout the model.

The populations are then used to reconstruct detailed line profiles through ray-tracing, which can be convolved with any beam or area. It can also be used to make velocity maps of the source. The adopted spectral resolution is $0.05 \mathrm{~km} \mathrm{~s}^{-1}$. RATRAN was benchmarked against many codes (van Zadelhoff et al. 2002). Furthermore, it was part of a recent benchmark test ${ }^{3}$ for line modeling of $\mathrm{H}_{2} \mathrm{O}$ (van der Tak et al. 2005).

Although level populations were calculated for all levels up to $E_{\text {up }}=2000 \mathrm{~K}$, only lines with an upper energy level below $500 \mathrm{~K}$ were selected for the presentation of results (see Table 3). Both $\mathrm{H}_{2} \mathrm{O}$ and $\mathrm{H}_{2}^{18} \mathrm{O}$ were modeled with a focus on lines that fall into the Herschel wavelength range. The ${ }^{16} \mathrm{O}:{ }^{18} \mathrm{O}$ isotope ratio was assumed to be 550:1, the ratio in the local ISM (Wilson \& Rood 1994). All generic models are placed at $150 \mathrm{pc}$, the typical distance of most nearby low-mass starforming regions such as Ophiuchus, Taurus, and Chameleon. Results were

3 See http://www. sron.rug.nl/ vdtak/H2O/ convolved with the appropriate Herschel beams and given in main beam temperatures. For reference, the typical sensitivities expected with HIFI $\left(5 \sigma, 0.5 \mathrm{~h}, 0.5 \mathrm{~km} \mathrm{~s}^{-1}\right.$ resolution) are $60 \mathrm{mK}$ for $557 \mathrm{GHz}, 180 \mathrm{mK}$ at $1130 \mathrm{GHz}$, and $600 \mathrm{mK}$ at $1669 \mathrm{GHz}$. For PACS, limiting line fluxes $(5 \sigma, 0.5 \mathrm{~h}$, point source) range from $6 \times 10^{-18} \mathrm{~W} \mathrm{~m}^{-2}$ to $35 \times 10^{-18} \mathrm{~W} \mathrm{~m}^{-2} 4$. To convert from main beam temperatures $T_{\mathrm{MB}}$ to fluxes $S_{v}$ in Jy $\left(10^{-26} \mathrm{~W} \mathrm{~m}^{-2} \mathrm{~Hz}^{-1}\right)$, use the formula

$S_{v}=2.65 \times 10^{8} \times T_{\mathrm{MB}}(\mathrm{K}) \times \theta_{0}^{2}\left({ }^{\prime}\right) \times \lambda^{-2}(\mu \mathrm{m})$.

This assumes a source size equivalent to the beam size. For sources smaller than the beam, a correction needs to be taken into account. The Herschel beam $\theta_{0}$ ranges from $40^{\prime \prime}\left(0.66^{\prime}\right)$ at $557 \mathrm{GHz}(539 \mu \mathrm{m})$ to $7^{\prime \prime}\left(0.12^{\prime}\right)$ at $3000 \mathrm{GHz}(100 \mu \mathrm{m})$ (see Table 3).

\section{Example: L 483}

\subsection{Physical structure}

As an illustrative model, the isolated Class 0 source L 483, (Motte \& André 2001) located at 200 pc, was modeled to investigate the sensitivity of the water line intensities to different abundances. This source has been studied extensively by Jørgensen et al. (2002) and Jørgensen (2004) and its physical and chemical structure is well-constrained through continuum and line data from (sub)millimeter single-dish telescopes and interferometers. The well-determined envelope and source parameters $\left(L_{\text {bol }}=9 L_{\odot}\right.$ and $\left.n_{0}\left(\mathrm{H}_{2}\right)=1 \times 10^{6} \mathrm{~cm}^{-3}\right)$ are roughly in the middle of our parameter range, making $\mathrm{L} 483$ an excellent test-case for modeling water emission. Only the inferred density slope, $p=0.9$, is somewhat flatter than for other sources, but this does not influence the model trends. For CO, a jump abundance at an evaporation temperature of $T \approx 30 \mathrm{~K}$ was found to explain the observed molecular lines. Any compact disk is constrained to a mass of $0.012 M_{\odot}$ using interferometer observations with the Sub-Millimeter Array (Jørgensen et al. 2007), negligible compared to the total estimated envelope mass of $4.4 M_{\odot}$ out to the $10 \mathrm{~K}$ radius of $10000 \mathrm{AU}$. In addition, this source has been observed with ISO-LWS (Giannini et al. 2001). The best-fit parameters from the dust radiative transfer $(Y=1400, p=0.9$, $\tau_{100}=0.3$ ), which fit both the SED and the spatial extent of the SCUBA continuum data, determine the physical structure of the envelope. $R_{\text {in }}$ (at $T=250 \mathrm{~K}$ ) is $9.9 \mathrm{AU}$ for this rather shallow density profile, and $R_{\text {out }}$ is $\sim 10000 \mathrm{AU}$. The radius where water freezes out onto the grains $(T=100 \mathrm{~K})$ is located at $35 \mathrm{AU}$. This L 483 model was used to explore a wider variety of water abundances than the generic models. Specifically, values of $1 \times 10^{-4}, 5 \times 10^{-5}, 1 \times 10^{-5}, 5 \times 10^{-6}$, and $1 \times 10^{-6}$ were used for $X_{0}$ and $1 \times 10^{-6}, 5 \times 10^{-7}, 1 \times 10^{-7}, 5 \times 10^{-8}$, and $1 \times 10^{-8}$ for $X_{\mathrm{d}}$. All other parameters were kept fixed. Ortho- and parawater were modeled separately, and water abundances are given for ortho- or para-water individually instead of the combined total water abundance with the used 3:1 ortho to para ratio. This allows independent probes of the ortho to para ratio. Thus, $X_{0}$ and $X_{\mathrm{d}}$ refer to either ortho- or para- $\mathrm{H}_{2} \mathrm{O}$, depending on the line in question.

\subsection{Results}

In Table 4 the integrated emission of both $\mathrm{H}_{2}^{16} \mathrm{O}$ and $\mathrm{H}_{2}^{18} \mathrm{O}$ lines, observable with Herschel-HIFI (see Table 3), are presented for

\footnotetext{
${ }^{4}$ Calculated with Hspot, see http://herschel.esac. esa.int/
} 
Table 4. Integrated intensities, $\int T_{\mathrm{MB}} \mathrm{d} V\left[\mathrm{~K} \mathrm{~km} \mathrm{~s}^{-1}\right]$, for $\mathrm{H}_{2} \mathrm{O}$ and $\mathrm{H}_{2}^{18} \mathrm{O}$ lines for $\mathrm{L} 483$ models with various abundances. Negative values indicate strong (self-) absorbed lines.

\begin{tabular}{|c|c|c|c|c|c|c|c|c|c|}
\hline Transition & & $X_{0}=10^{-4}$ & & & $X_{0}=10^{-5}$ & & & $X_{0}=10^{-6}$ & \\
\hline$X_{\mathrm{d}}=$ & $10^{-6}$ & $10^{-7}$ & $10^{-8}$ & $10^{-6}$ & $10^{-7}$ & $10^{-8}$ & $10^{-6}$ & $10^{-7}$ & $10^{-8}$ \\
\hline \multicolumn{10}{|c|}{ Ortho- $\mathrm{H}_{2} \mathrm{O}$ transitions } \\
\hline $1_{10}-1_{01}$ & 2.7 & 2.4 & 0.83 & 2.8 & 1.0 & $8.8(-2)$ & 9.5 & 3.8 & 0.57 \\
\hline $2_{12}-1_{01}$ & -0.2 & -0.13 & 0.27 & -0.35 & -0.38 & -0.33 & 3.0 & 0.23 & -0.05 \\
\hline $2_{21}-2_{12}$ & 1.7 & 0.27 & 0.20 & 0.17 & 0.32 & 0.11 & 1.7 & 0.37 & 0.12 \\
\hline $3_{12}-2_{21}$ & 0.8 & 0.17 & 0.10 & 0.47 & 0.12 & $7.8(-2)$ & 0.95 & 0.20 & 7.1(-2) \\
\hline $3_{12}-3_{03}$ & 0.8 & 0.29 & 0.13 & 0.43 & 0.22 & $6.0(-2)$ & 0.94 & 0.39 & $7.4(-2)$ \\
\hline $3_{21}-3_{12}$ & 1.3 & 0.35 & 0.11 & 0.73 & 0.26 & $5.4(-2)$ & 1.5 & 0.46 & $8.2(-2)$ \\
\hline \multicolumn{10}{|c|}{ Para- $\mathrm{H}_{2} \mathrm{O}$ transitions } \\
\hline $1_{11}-0_{00}$ & $-6.5(-3)$ & 1.23 & -0.17 & -0.12 & $5.2(-2)$ & 0.29 & 3.12 & 1.4 & -0.13 \\
\hline $202-1_{11}$ & 1.1 & 0.59 & 0.26 & 1.4 & 0.6 & 0.45 & 0.6 & 1.4 & 0.26 \\
\hline $2_{11}-2_{02}$ & 0.85 & 0.73 & 0.16 & 0.99 & 0.6 & 0.34 & 0.96 & 1.1 & 0.15 \\
\hline $2_{20}-2_{11}$ & 0.38 & 0.61 & 0.18 & 0.45 & 0.50 & 0.31 & 0.38 & 0.83 & 0.13 \\
\hline $3_{31}-4_{04}$ & $4.4(-2)$ & $6.3(-2)$ & $6.3(-2)$ & $1.9(-2)$ & $1.9(-2)$ & $2.7(-2)$ & $3.7(-3)$ & $1.9(-3)$ & $1.3(-3)$ \\
\hline $4_{22}-3_{31}$ & $5.0(-2)$ & $6.8(-2)$ & $3.7(-2)$ & $5.2(-2)$ & $5.6(-2)$ & $5.1(-2)$ & $1.8(-2)$ & $1.5(-2)$ & $9.7(-3)$ \\
\hline $4_{22}-4_{13}$ & 0.21 & $8.6(-2)$ & $5.3(-2)$ & 0.25 & $8.3(-2)$ & $8.0(-2)$ & 0.24 & 0.10 & $5.8(-3)$ \\
\hline $5_{24}-4_{31}$ & $6.0(-2)$ & $8.8(-2)$ & $5.5(-2)$ & $3.2(-2)$ & $4.3(-2)$ & $5.5(-2)$ & $4.0(-3)$ & $5.9(-3)$ & $3.3(-3)$ \\
\hline \multicolumn{10}{|c|}{ Ortho- $\mathrm{H}_{2}^{18} \mathrm{O}$ transitions } \\
\hline $1_{10}-1_{01}$ & $-3.3(-2)$ & $-5.6(-3)$ & $2.3(-2)$ & $-4.0(-2)$ & $-2.0(-2)$ & $5.2(-3)$ & $-1.9(-2)$ & $-2.4(-2)$ & $-3.9(-4)$ \\
\hline $2_{12}-1_{01}$ & -0.13 & $-3.3(-2)$ & $1.2(-2)$ & -0.13 & $-4.5(-2)$ & $-6.7(-4)$ & -0.12 & $-4.9(-2)$ & $-5.2(-3)$ \\
\hline $2_{21}-2_{12}$ & $4.1(-3)$ & $4.2(-3)$ & $3.7(-3)$ & $1.8(-3)$ & $9.6(-4)$ & $9.3(-4)$ & $1.0(-3)$ & $1.3(-4)$ & $1.1(-4)$ \\
\hline $3_{12}-2_{21}$ & $5.6(-2)$ & $6.0(-2)$ & $5.9(-2)$ & $3.6(-3)$ & $3.0(-3)$ & $3.0(-3)$ & $5.9(-4)$ & $1.39(-4)$ & $1.2(-4)$ \\
\hline $3_{12}-3_{03}$ & $2.5(-2)$ & $2.6(-2)$ & $2.5(-2)$ & $8.5(-3)$ & $6.9(-3)$ & $6.8(-3)$ & $2.0(-3)$ & $4.6(-4)$ & $3.8(-4)$ \\
\hline $3_{21}-3_{12}$ & $1.7(-2)$ & $1.9(-2)$ & $1.8(-2)$ & $4.5(-3)$ & $3.6(-3)$ & $3.5(-3)$ & $1.0(-3)$ & $2.1(-4)$ & $1.7(-4)$ \\
\hline \multicolumn{10}{|c|}{ Para- $\mathrm{H}_{2}^{18} \mathrm{O}$ transitions } \\
\hline $1_{11}-0_{00}$ & -0.21 & $-7.7(-2)$ & $8.7(-3)$ & -0.16 & $-8.8(-2)$ & $-4.7(-3)$ & -0.21 & $-9.3(-2)$ & $-1.2(-2)$ \\
\hline $2_{02}-1_{11}$ & $5.3(-2)$ & $4.0(-2)$ & $3.8(-2)$ & $4.4(-2)$ & $2.0(-2)$ & $1.8(-2)$ & $2.5(-2)$ & $4.7(-3)$ & $3.1(-3)$ \\
\hline $2_{11}-2_{02}$ & $2.5(-2)$ & $1.9(-2)$ & $1.9(-2)$ & $2.0(-2)$ & $7.9(-3)$ & $7.6(-3)$ & $9.6(-3)$ & $1.5(-3)$ & $1.1(-3)$ \\
\hline $2_{20}-2_{11}$ & $1.7(-2)$ & $1.4(-2)$ & $1.4(-2)$ & $1.6(-2)$ & $6.5(-3)$ & $6.2(-3)$ & $7.2(-3)$ & $1.0(-3)$ & $6.9(-4)$ \\
\hline $3_{31}-4_{04}$ & $9.2(-5)$ & $1.2(-4)$ & $1.2(-4)$ & $5.7(-6)$ & $4.6(-6)$ & $4.6(-6)$ & $5.1(-7)$ & $2.5(-7)$ & $2.3(-7)$ \\
\hline $4_{22}-3_{31}$ & $6.3(-4)$ & $8.4(-4)$ & $8.5(-4)$ & $2.8(-5)$ & $2.3(-5)$ & $2.3(-5)$ & $9.1(-7)$ & $6.7(-7)$ & $6.4(-7)$ \\
\hline $4_{22}-4_{13}$ & $2.5(-2)$ & $3.0(-2)$ & $3.0(-2)$ & $1.5(-3)$ & $1.2(-3)$ & $1.2(-3)$ & $4.8(-5)$ & $3.5(-5)$ & $3.4(-5)$ \\
\hline $5_{24}-4_{31}$ & $7.1(-5)$ & $1.1(-4)$ & $1.1(-4)$ & $1.6(-6)$ & $1.3(-6)$ & $1.3(-6)$ & $4.6(-8)$ & 4.2(-8) & $4.0(-8)$ \\
\hline
\end{tabular}

In this and subsequent tables, the notation $A(B)$ indicates $A \times 10^{B}$.

the reference grid of $\mathrm{L} 483$, whereas Table 5 presents the peak temperatures. Detailed contour plots of all HIFI lines as functions of $X_{0}$ and $X_{\mathrm{d}}$ are presented in Fig. 2 the online appendix. Many of the trends discussed below are similar to those found for AFGL 2591, as discussed in Poelman \& van der Tak (2007). Overall, lines are found to belong to three categories. First, all excited $\mathrm{H}_{2}^{18} \mathrm{O}$ and several $\mathrm{H}_{2} \mathrm{O}$ highly excited $\left(E_{\text {up }}>200 \mathrm{~K}\right)$ lines are completely optically thin. They have Gaussian profiles with widths that depend on the turbulent width. Due to the freeze-out of water onto grains below $100 \mathrm{~K}$, these optically thin lines probe the inner warm dense region. For the model where water has a constant abundance of $10^{-6}$, emission from the warm dense inner region still dominates and is optically thin (either due to the low population of the highly excited states or the low abundance of $\mathrm{H}_{2}^{18} \mathrm{O}$ ). A good example is the $\mathrm{H}_{2} \mathrm{O} 5_{24}-4_{31}$ $970 \mathrm{GHz}$ line shown in Fig. 2 (right).

The ground state lines of both $\mathrm{H}_{2}^{18} \mathrm{O}$ and $\mathrm{H}_{2} \mathrm{O}$, together with most $\mathrm{H}_{2} \mathrm{O}$ excited lines with energies below $200 \mathrm{~K}$, provide a strong contrast with the optically thin lines. These lines only probe the outer region. Even an abundance of water as low as $10^{-8}$ in the cold outer envelope produces a high enough column for these lines to become optically thick. The extremely high optical depth $(\tau>25)$ and the absorptions into the dust further prevent the integrated intensity from probing the entire envelope. Thus, these lines trace the outer abundance $X_{\mathrm{d}}$, and self-absorption and absorption of the dust continuum are commonly found in their line profiles. However, most optically thick lines have wings that are either optically thin or less optically thick. These wings may be able to probe the inner region up to the boundary layer around $T=100 \mathrm{~K}$. See the $1_{10}-1_{01}$ line in Fig. 2 (left), Table 5 and the spectra in Figs. 3 and 4 (discussed in Sect. 3.4).

Finally, some lines are optically thick for a high $X_{0}-X_{\mathrm{d}}$ combination, but are optically thin at low abundances. It is not known a-priori if these lines originate within the cold outer envelope or the warm dense inner regions. Examples are several of the highly excited $\mathrm{H}_{2} \mathrm{O}$ lines, but such dependencies are also seen for lowly excited $\mathrm{H}_{2}^{18} \mathrm{O}$ lines. Figure 2 (middle) shows an example with the $\mathrm{p}-\mathrm{H}_{2}^{18} \mathrm{O} 2_{02}-1_{11}$ line. It can be seen that, depending on the total column of water, this line either traces $X_{\mathrm{d}}$ at high columns or $X_{0}$ at lower columns. For the $\mathrm{H}_{2}^{18} \mathrm{O}$ lines, a clear dividing line between dependency on $X_{0}$ or $X_{\mathrm{d}}$ lies at $X_{\mathrm{d}}\left(\mathrm{H}_{2}^{18} \mathrm{O}\right)<10^{-9.5}$ (see online appendix). Such a division is less clear for $\mathrm{H}_{2}^{16} \mathrm{O}$. The dust can become optically thick, especially at high frequencies, so the higher frequency lines will not be able to probe the inner region at all. For the $\mathrm{L} 483$ models, this only takes place at frequencies higher than $1500 \mathrm{GHz}$, but see Sect. 5 for further discussion. In the following, individual $\mathrm{H}_{2} \mathrm{O}$ and $\mathrm{H}_{2}^{18} \mathrm{O}$ lines are discussed in more detail. 
Table 5. Peak temperatures $[\mathrm{K}]$ of $\mathrm{H}_{2} \mathrm{O}$ lines for $\mathrm{L} 483$ models of various abundances. Negative values primarily indicate absorption into the dust continuum.

\begin{tabular}{|c|c|c|c|c|c|c|c|c|c|}
\hline \multirow{2}{*}{$\begin{array}{c}\text { Transition } \\
X_{\mathrm{d}}=\end{array}$} & \multicolumn{3}{|c|}{$X_{0}=10^{-4}$} & \multicolumn{3}{|c|}{$X_{0}=10^{-5}$} & \multicolumn{3}{|c|}{$X_{0}=10^{-6}$} \\
\hline & $10^{-6}$ & $10^{-7}$ & $10^{-8}$ & $10^{-6}$ & $10^{-7}$ & $10^{-8}$ & $10^{-6}$ & $10^{-7}$ & $10^{-8}$ \\
\hline \multicolumn{10}{|c|}{ Ortho- $\mathrm{H}_{2} \mathrm{O}$ transitions } \\
\hline $1_{10}-1_{01}$ & 2.8 & 2.1 & 0.65 & 2.8 & 1.1 & 0.2 & 5.4 & 2.8 & 0.5 \\
\hline $2_{12}-1_{01}$ & 0.45 & 0.35 & 0.25 & 0.3 & -0.13 & -0.13 & 1.9 & -0.13 & 0.25 \\
\hline $2_{21}-2_{12}$ & 1.1 & 0.1 & 0.05 & 0.2 & 0.1 & $3.8(-2)$ & 1.6 & 0.21 & $4.5(-2)$ \\
\hline $3_{12}-2_{21}$ & 0.28 & $6.5(-2)$ & $2.4(-2)$ & 0.18 & $4.5(-2)$ & $2.1(-2)$ & 0.36 & 0.1 & $2.4(-2)$ \\
\hline $3_{12}-3_{03}$ & 0.27 & 0.12 & 4.1(-2) & 0.12 & 0.1 & $1.5(-2)$ & 0.4 & 0.17 & $2.8(-2)$ \\
\hline $3_{21}-3_{12}$ & 0.5 & 0.18 & 4.1(-2) & 0.33 & 0.13 & $1.5(-2)$ & 0.55 & 0.26 & $3.2(-2)$ \\
\hline \multicolumn{10}{|c|}{ Para- $\mathrm{H}_{2} \mathrm{O}$ transitions } \\
\hline $1_{11}-0_{00}$ & 1.5 & 1.3 & $-9(-2)$ & 0.45 & 0.45 & 0.45 & 1.6 & 1.25 & 0.13 \\
\hline $2_{02}-1_{11}$ & 1.6 & 0.55 & $8(-2)$ & 1.1 & 0.4 & 0.18 & 0.9 & 1.0 & $8(-2)$ \\
\hline $22_{11}-2_{02}$ & 0.8 & 0.22 & $7(-2)$ & 0.38 & 0.18 & 0.16 & 0.45 & 0.38 & $7.5(-2)$ \\
\hline $2_{20}-2_{11}$ & 0.4 & 0.28 & $7.5(-2)$ & 0.12 & 0.24 & 0.17 & 0.1 & 0.35 & $7(-2)$ \\
\hline $3_{31}-4_{04}$ & $2.8(-2)$ & $2.2(-2)$ & $2.2(-2)$ & $1(-2)$ & $1(-2)$ & $1.9(-2)$ & $2.5(-3)$ & $1.5(-3)$ & $8(-4)$ \\
\hline $4_{22}-3_{31}$ & $2.8(-2)$ & $2(-2)$ & $1(-2)$ & $2.3(-2)$ & $2.4(-2)$ & $1.5(-2)$ & $1.2(-2)$ & $1(-2)$ & $6(-3)$ \\
\hline $4_{22}-4_{13}$ & 0.16 & $2.4(-2)$ & $1.4(-2)$ & 0.12 & $2.7(-2)$ & $1.7(-2)$ & 0.12 & $4.5(-2)$ & $2.2(-2)$ \\
\hline $5_{24}-4_{31}$ & $2.2(-2)$ & $2.6(-2)$ & $1.6(-2)$ & $1.8(-2)$ & $1.9(-2)$ & $2.4(-2)$ & $2.5(-3)$ & $3.5(-3)$ & $2(-3)$ \\
\hline
\end{tabular}
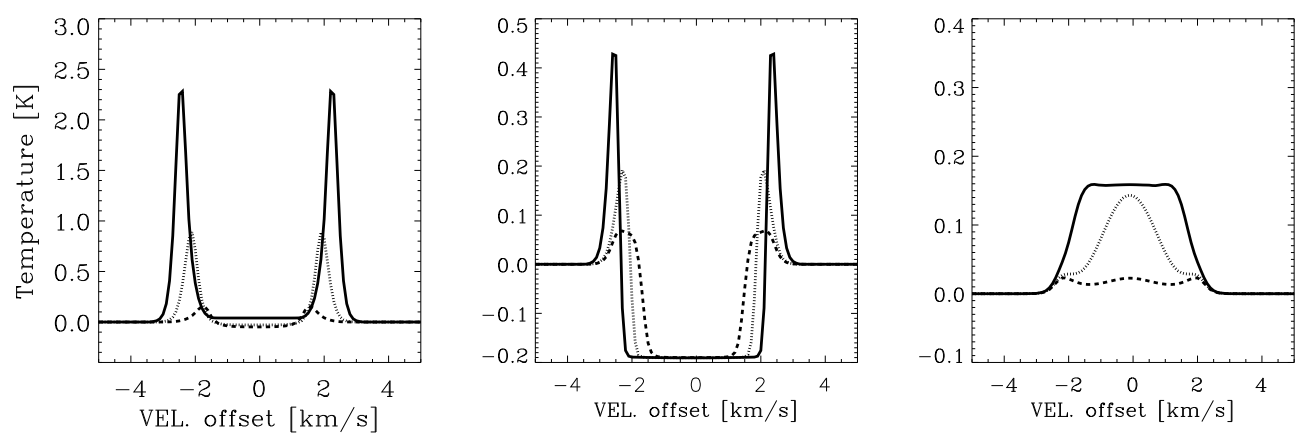

Fig. 3. $\mathrm{H}_{2} \mathrm{O}$ spectra for an abundance $X_{0}$ of $10^{-5}$ for the $1_{10}-1_{01}(557 \mathrm{GHz}$, left $), 2_{12}-1_{01}\left(1669 \mathrm{GHz}\right.$ middle), and $3_{12}-3_{03}(1097 \mathrm{GHz}$ right) lines. Lines are shown for $X_{\mathrm{d}}$ of $10^{-6}$ (solid), $10^{-7}$ (dot), and $10^{-8}$ (dash).
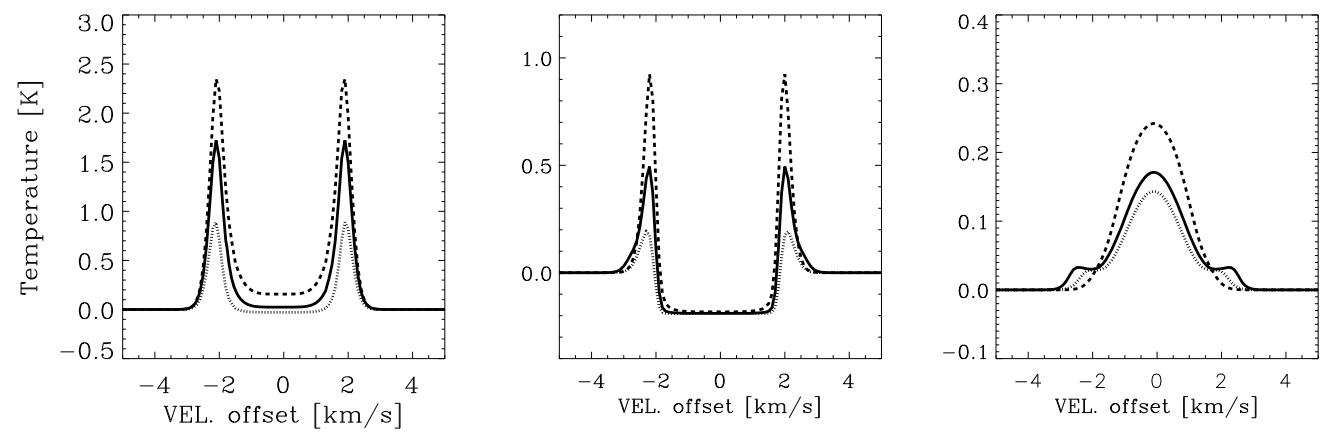

Fig. 4. $\mathrm{H}_{2} \mathrm{O}$ spectra for an abundance $X_{\mathrm{d}}$ of $10^{-7}$ for the $1_{10}-1_{01}(557 \mathrm{GHz}$ left $), 2_{12}-1_{01}\left(1669 \mathrm{GHz}\right.$ middle), and $3_{12}-3_{03}(1097 \mathrm{GHz}$ right $)$ lines. Lines are shown for $X_{0}$ of $10^{-4}$ (solid), $10^{-5}$ (dot), and $10^{-6}$ (dash).

\subsection{Integrated emission}

\subsection{1. $\mathrm{H}_{2} \mathrm{O}$}

The main isotopologue of water, $\mathrm{H}_{2}^{16} \mathrm{O}$, is optically thick in most transitions considered here. All lines with $J_{\text {up }}=1$ and 2 are optically thick and trace the outer cold envelope. Most other lines trace either the warm inner or cold outer envelope. The integrated intensities of these "hybrid" lines depend sensitively on the precise abundance parameters of the L 483 reference model. In particular, the radius where the line turns optically thick is comparable to that where the one where the temperature reaches
$100 \mathrm{~K}$ for this model. These are the $2_{21}-2_{12}, 3_{12}-2_{21}, 3_{12}-3_{03}$, $3_{21}-3_{12}$ and $4_{22}-4_{13}$ lines. At low abundances of $X_{\mathrm{d}}$, they depend more on $X_{0}$. If the abundance in the outer region is larger than $5 \times 10^{-8}$, they depend on $X_{\mathrm{d}}$ instead. The integrated intensities range from several hundreds of $\mathrm{mK} \mathrm{km} \mathrm{s}^{-1}$ in absorption to a few $\mathrm{K} \mathrm{km} \mathrm{s}^{-1}$ in emission, with peak brightness from $100 \mathrm{mK}$ to $1 \mathrm{~K}$, readily detectable with HIFI. The group of highly excited para-lines have peaks up to a few tens of $\mathrm{mK}$, requiring much longer integration times.

Dependencies of the line strengths on abundances are not linear. An exception is whenever the line is completely optically 
thin over the entire profile. This is not the case for most lines and parts of the line profile will be optically thin and parts optically thick, causing complicated dependencies. For instance, the total integrated intensity of the $557 \mathrm{GHz}$ line depends sensitively on the inner and outer abundances. Abundance combinations of $10^{-4} / 10^{-6}$ produce lines with less total emission than $10^{-6} / 10^{-6}$. The $10^{-6} / 10^{-6}$ combination contains less water emission originating from the warm inner regions, but more dust continuum is able to escape the inner regions in the $10^{-6} / 10^{-6}$. This influences the population distribution in the outer region, resulting in less central absorption. Since absorption is a major feature within this line profile, the total integrated line intensity effectively goes up.

\subsection{2. $\mathrm{H}_{2}^{18} \mathrm{O}$}

Because of the low abundance of the $\mathrm{H}_{2}^{18} \mathrm{O}$ isotopologue, most of its lines are optically thin. Only the three transitions connecting to the ground levels of ortho- and para- $\mathrm{H}_{2}^{18} \mathrm{O}-1_{11}-0_{00}, 1_{10}-1_{01}$, and $2{ }_{12}-1_{01}-$ have optical depths higher than 5 for all abundances. However, excited lines with $J_{\text {up }}=2$ are optically thick in their line centers for $X_{0}>10^{-5}$. The integrated intensities of these $\mathrm{H}_{2}^{18} \mathrm{O}$ lines range from a few hundred $\mathrm{mK} \mathrm{km} \mathrm{s}{ }^{-1}$ in absorption to a few tens of $\mathrm{mK} \mathrm{km} \mathrm{s}^{-1}$ in emission, whereas peak temperatures range from a few $\mathrm{mK}$ to a few tens of $\mathrm{mK}$, thus requiring long integration times. Due to the low abundance, the dependence on either $X_{0}$ or $X_{\mathrm{d}}$ is much more evident than for $\mathrm{H}_{2}^{16} \mathrm{O}$ (see Fig. 2 for example). The optically thick ground-state lines accurately trace $X_{\mathrm{d}}$, while optically thin excited lines only depend on $X_{0}$. The few optically thick excited lines trace $X_{0}$ as well, since the $\tau=1$ surface lies within the $100 \mathrm{~K}$ radius. Higher excitation $\mathrm{H}_{2}^{18} \mathrm{O}$ lines are not very strong, in the range of a few micro-K to a few milli-K, too weak to be detectable with HIFI in reasonable integration times.

\subsection{Line profiles}

A number of characteristic line profiles are presented in Figs. 3 and 4 . The continuum has been subtracted so that the profiles can be readily compared on the same scale. The total profile contains valuable information about the envelope structure and velocity profile, none of which can be determined using the integrated line intensity alone. As seen in the examples, there is a wide variety of model profiles depending on a number of parameters. First, the excitation energy of the upper state, the total water column density and the critical density of the line in question will determine whether an emission line is optically thick, thin or a combination of both. Most lines show a combination, with optically thin wings around an optically thick line center. Second, the width depends on the systematic and turbulent velocity. Third, water in the colder outer envelope will absorb photons and scatter the radiation, causing an absorption feature. Finally, the optical depth of dust itself blocks line emission at frequencies above $1500 \mathrm{GHz}$ originating in the inner region. In the following these profile shapes are discussed in more detail.

The profiles of optically thin lines are Gaussian and are good tracers of the velocity profile. Optically thin lines always trace the inner warm region, where the higher excitation states are populated. The ratio of the line wing over the peak temperature can vary, depending on the excitation energy of the line. Higher excitation lines are broader, while low excitation lines have a narrower peak with very weak line wings. An example in Fig. 4 is the $3_{12}-3_{03} 1097 \mathrm{GHz}$ line, which is completely optically thin for $X_{0}=10^{-6}$. The line center becomes optically thick at $X_{0}=10^{-5}$ and thus decreases, but the optically thin line wings are noticeably higher in emission. At $X_{0}=10^{-4}$, the optically thick line center is brighter than at $10^{-5}$ due to the high abundance, but still weaker than the optically thin emission at $X_{0}=10^{-6}$.

The profiles of optically thick lines are much more complicated. Their wings can either be optically thick or thin, depending on the abundances and frequency of the line. This gives rise to three different profiles.

- Lines with a Gaussian line center, but with enhanced line wings. A characteristic "bump" can be seen at velocities where the line profile changes from optically thick to thin when going to more extreme velocities. The line center is optically thick, but is heavily pumped by the line radiation coming from water in the warmer regions further inwards in the envelope. This behavior is often called "effectively optically thin". Such lines trace the inner abundance $X_{0}$, unless optically thick dust veils the inner region. An example is seen by the $3_{12}-3_{03} 1097 \mathrm{GHz}$ line with an $X_{0}$ of $10^{-4}$ in Fig. 4.

- "Flat top" lines, where the center of the line is optically thick, but not self-absorbed. The center of the line is dominated by emission from a small region with a specific temperature. Line wings are optically thin. Material at larger radii is unable to cause self-absorption, and there is not enough material deeper within the envelope to make such a line "effectively optically thin". Optically thick dust can prevent warmer regions from influencing the line at higher frequencies. An example is given by the $3_{12}-3_{03} 1097 \mathrm{GHz}$ line with $X_{\mathrm{d}}=10^{-6}$ in Fig. 3.

- Deeply (self)-absorbed lines with most of the emission in the line wings. Absorption into the dust occurs, and is stronger for high frequency lines, where the dust continuum is higher. All three ground state lines show such profiles. Examples are the $1_{10}-1_{01}$ and $2_{12}-1_{01}$ lines in Figs. 3 and 4 . Both the depth and the width of the absorption vary, as do the line wing strengths:

(i) Absorption depth: if dust is optically thick (e.g., at $1669 \mathrm{GHz}$ ), the depth is independent of both $X_{0}$ and $X_{\mathrm{d}}$ as long as $\tau \gg 1$, and only depends on the radius where the dust turns optically thick, setting the continuum level. In Figs. 3 and 4 (middle) the $1669 \mathrm{GHz}$ continuum-subtracted line is always at $-0.2 \mathrm{~K}$. If dust is optically thin (e.g., the $557 \mathrm{GHz}$ $1_{10}-1_{01}$ line), the depth $v$ of the absorption depends on both $X_{\mathrm{d}}$ and $X_{0}$ and is caused by self-absorption of the water in the cold outer region against the warm emission. Indeed, it is found that water at around $70-90 \mathrm{~K}$ acts as a warm background emitter and water at $20 \mathrm{~K}$ as the cold absorber.

(ii) Absorption width: again there is a difference between high and low optical depths of dust. For $1669 \mathrm{GHz}$ the width of the absorption depends on $X_{\mathrm{d}}$, but not on $X_{0}$. For $557 \mathrm{GHz}$, there is no dependence on abundance. The width of the $a b-$ sorption is the same for varying $X_{0}$ (the temperature at which the water emits changes little), and even though the profiles appear different for different $X_{\mathrm{d}}$, this can be attributed to the changing absorption depth, not width.

(iii) Line wing strength: the strength of the line wing, which often contains the bulk of the line emission, is determined by the optical depth of the dust and gas. Most often, the peaks seen in the wings of the ground-state lines are still optically thick. The wings trace both $X_{\mathrm{d}}$ (see $1_{10}-1_{01}$ and $2_{12}-1_{01}$ lines in Fig. 3, left, middle), which still contain large columns of the gas, and $X_{0}\left(1_{10}-1_{01}\right.$ and $2_{12}-1_{01}$ Fig. 4, left, middle), although in that case the relation is not straightforward. Line 
pumping from the warm gas further inwards is the main origin of the dependency from $X_{0}=10^{-4}$ to $10^{-5}$. However, if $X_{\mathrm{d}}$ and $X_{0}$ are low enough, the line wings turn optically thin and are significantly enhanced (Fig. 3) because higher temperatures are probed. This behavior is seen for a $X_{\mathrm{d}} / X_{0}$ combination of $10^{-7} / 10^{-6}$ where the line wings are brighter than for $X_{\mathrm{d}} / X_{0}$ combinations of $10^{-7} / 10^{-4}$ and $10^{-7} / 10^{-5}$.

\subsection{HIFI diagnostic lines}

Although the beams for HIFI and the accompanying dilution factors are large compared to the physical size of the warm gas (see Table 3), the inner warm region is detectable through the higher excited states. The outer cold envelope is completely transparent for these lines. Considering the expected sensitivities of HIFI, a combination of different lines can constrain the water abundance profile. The $\mathrm{H}_{2}^{18} \mathrm{O} 2_{02}-1_{11} 994 \mathrm{GHz}$ line in HIFI Band 4 is mostly optically thin and traces $X_{0}$, except at $\mathrm{H}_{2}^{16} \mathrm{O} X_{\mathrm{d}}$ abundances higher than $5 \times 10^{-6}$. However, the line is weak (a few tens of $\mathrm{mK}$ ) and needs at least a few hours of integration to be detected. A possible alternative is the high excitation $\mathrm{H}_{2}^{16} \mathrm{O} 3_{12}-3_{03}$ line in HIFI Band 4, which can be detected in roughly an hour of integration. It traces $X_{0}$ at high $X_{0}$ abundances, but for $X_{0}$ lower than $10^{-5}$, emission coming from the colder outer part dominates, thus tracing $X_{\mathrm{d}}$. This emission originates in the region with temperatures ranging from $70-100 \mathrm{~K}$. Once the inner abundance $X_{0}$ is well-constrained, ground-state lines can in turn be used to constrain the abundance in the outer region. The bright wings of the $557 \mathrm{GHz}$ line (HIFI Band 1) are detectable within a few minutes, but the entire line profile is needed for proper analysis (see Sect. 3.4). The dependence on $X_{\mathrm{d}}$ can be traced most accurately using the line profiles of the $1_{11}-0_{00} 1113 \mathrm{GHz}$ para- $\mathrm{H}_{2} \mathrm{O}$ and $1101 \mathrm{GHz}$ para- $\mathrm{H}_{2}^{18} \mathrm{O}$ lines in Band 4, but resolving these profiles requires much longer integration times than the $557 \mathrm{GHz}$ line. The $1667 \mathrm{GHz} 2_{12}-1_{01}$ ortho- $\mathrm{H}_{2} \mathrm{O}$ line in Band 6 is intrinsically brighter, but will take almost an hour of integration due to the low sensitivity of HIFI at this frequency. In addition, dust can become optically thick at this high frequency so that the line profile only traces the structure in the outer envelope.

The optically thick $\mathrm{H}_{2}^{16} \mathrm{O} 2_{02}-1_{11} 988 \mathrm{GHz}$ line, with its optically thin $\mathrm{H}_{2}^{18} \mathrm{O}$ counterpart are the best candidates for determining the optical depth of water, but the $\mathrm{H}_{2}^{18} \mathrm{O}$ lines require significant integration time (see above). An alternative is provided by comparison between of the $548 \mathrm{GHz} \mathrm{H}{ }_{2}^{18} \mathrm{O}$ and $557 \mathrm{GHz}$ $\mathrm{H}_{2}^{16} \mathrm{O}$ ground-state lines, but $\mathrm{H}_{2}^{18} \mathrm{O}$ is still optically thick so that detailed modeling is required. The $1_{11}-0_{00} 1113 \mathrm{GHz}, 2_{02}-1_{11}$ $988 \mathrm{GHz}$, and $2_{11}-2_{02} 752 \mathrm{GHz}$ para- $\mathrm{H}_{2} \mathrm{O}$ lines are well-suited to probing the physical structure of the envelope. Due to their different excitation energies and optical depths, these lines probe different environments within the protostellar envelope, and their line ratios are not affect by the uncertain ortho/para ratio.

\subsection{Comparison with ISO-LWS data}

The source L 483 was observed with ISO-LWS (Giannini et al. 2001). The para $2_{20}-1_{11}$ line at $2968 \mathrm{GHz}(101 \mu \mathrm{m})$ was detected with a flux of $8.6 \pm 1.0 \times 10^{-20} \mathrm{~W} \mathrm{~cm}^{-2}$ and the ortho $2_{12}-1_{01}$ line at $1670 \mathrm{GHz}(179.5 \mu \mathrm{m})$ was found to have an upper limit of $4.3 \times 10^{-20} \mathrm{~W} \mathrm{~cm}^{-2}$. The low LWS resolving power of $\lambda / \Delta \lambda=$ $200\left(\sim 1500 \mathrm{~km} \mathrm{~s}^{-1}\right)$ was insufficient for determining the effect of any self-absorption or disentangling outflow components from quiescent envelope material. The best-fitting LVG model for the high- $J$ CO lines gave a component of gas with $T=850-1800 \mathrm{~K}$; it is expected that this hot gas contributes significantly to the water emission (Giannini et al. 2001).

The above models, corrected for the ISO beam of $80^{\prime \prime}$, are compared to the observed intensity. Our models show a strong absorption into the dust for both lines with optically thin line wings. Integrated intensities range from $-7 \times 10^{-22}$ up to $17 \times$ $10^{-20} \mathrm{~W} \mathrm{~cm}^{-2}$ for the $2_{20}-1_{11}$ line and from $0.9 \times 10^{-20} \mathrm{~W} \mathrm{~cm}^{-2}$ to $16 \times 10^{-20} \mathrm{~W} \mathrm{~cm}^{-2}$ for the ortho $2_{12}-1_{01}$ line for different $X_{0^{-}}$ $X_{\mathrm{d}}$ combinations. The best fit, using both the detection and upper limit, is found for an outer abundance of $10^{-7}$ and an inner abundance between $10^{-6}$ and $10^{-5}$. The detected line is reproduced within $30 \%$, while the upper limit is only $50 \%$ higher than the intensity produced by the model. For lower values of $X_{\mathrm{d}}$, around $10^{-8}$, the integrated emission in our model is almost an order of magnitude lower than the observed $2_{20}-1_{11}$ line. Such low outer abundances are found for cold regions in the envelopes around high-mass YSOs (Boonman et al. 2003), but appear inconsistent with the data for this low-mass YSO, unless the detected emission originates fully in the outflow. Higher inner abundances cannot be ruled out. However, the self-absorption in the models becomes stronger, resulting in a worse fit to the detected line and upper limit.

Other water lines within the ISO-LWS domain were not detected. The derived upper limits were found to be at least a factor 5 higher than fluxes predicted from all possible models. At short $(>80 \mu \mathrm{m})$ wavelengths, the difference is a few orders of magnitude. Since all lines are unresolved, no conclusion can be reached as to whether the detected water emission is dominated by outflow contributions or by thermal excitation within the circumstellar envelope with a high abundance. This example illustrates the need for spectrally and spatially resolved data of a number of lines to disentangle the different physical components.

\section{General parameter study}

Table 6 and Figs. 5 and 6 present the results for a grid probing the parameters from Table 1 for a constant abundance combination of $10^{-4}$ for $X_{0}$ and $10^{-7}$ for $X_{\mathrm{d}}$. Results for the full grid, including all probed abundances, can be found in the online appendix. The main variables of the grid - the luminosity, the density, and the density profile steepness - are discussed within this section. The dependence on abundance mirrors the behavior seen for the L 483 model discussed in Sect. 3. For most combinations of envelope parameters, the $\mathrm{H}_{2}^{16} \mathrm{O}$ transitions studied here are optically thick, sometimes with optically thin line wings. For $\mathrm{H}_{2}^{18} \mathrm{O}$ lines, most transitions are optically thin, although the three ground state lines are optically thick at line center. The only optically thin $\mathrm{H}_{2}^{16} \mathrm{O}$ lines are those for which the upper level of the transition has an excitation temperature above 200-250 K, specifically the lines with $J=3$ and higher. These lines are only optically thin if the densities are low enough, $<10^{7} \mathrm{~cm}^{-3}$, and abundances are low, $X_{0}<10^{-5}$ and $X_{\mathrm{d}}<10^{-7}$. For typical lowmass envelopes, such densities are only encountered within the inner region, where enhanced abundances are found. Thus, because many higher excited $\mathrm{H}_{2}^{16} \mathrm{O}$ lines become optically thick only in the very dense warm inner region, they are still good tracers of the inner region (see Sect. 3.3.1 and Fig. 4 right). For high densities and thus higher envelope masses, the larger dust column plays a major role by heavily influencing the water line emission. This is discussed in Sect. 5. 
Table 6. Integrated intensities, $\int T_{\mathrm{MB}} \Delta V\left[\mathrm{~K} \mathrm{~km} \mathrm{~s}^{-1}\right]$, for $\mathrm{H}_{2} \mathrm{O}$ lines in the wide parameter grid for an abundance combination of $X_{0}=10^{-4}$ and $X_{\mathrm{d}}=10^{-7}$.

\begin{tabular}{|c|c|c|c|c|c|c|}
\hline \multirow[t]{3}{*}{ Transition } & \multicolumn{6}{|c|}{ Integrated intensity $\left(\int T_{\mathrm{MB}} \Delta V\left[\mathrm{~K} \mathrm{~km} \mathrm{~s}^{-1}\right]\right)$} \\
\hline & \multicolumn{3}{|c|}{ Lum. (w. $\left.n_{0}=10^{6} \mathrm{~cm}^{-3}\right)$} & \multicolumn{3}{|c|}{ Dens. $\left(\right.$ w. $\left.L=7 L_{\odot}\right)$} \\
\hline & 2 & 7 & 25 & 0.4 & 1 & 5 \\
\hline \multicolumn{7}{|c|}{$p=1.5$} \\
\hline \multicolumn{7}{|c|}{ Ortho- $\mathrm{H}_{2} \mathrm{O}$ transitions } \\
\hline $1_{10}-1_{01}$ & 0.37 & 0.95 & 0.70 & 0.43 & 0.95 & 2.1 \\
\hline $2_{12}-1_{01}$ & 0.35 & 0.36 & -1.2 & 0.30 & 0.36 & -4.4 \\
\hline $2_{21}-2_{12}$ & 0.29 & 0.87 & 1.5 & 0.60 & 0.87 & 0.74 \\
\hline $3_{12}-3_{03}$ & 0.21 & 0.60 & 1.5 & 0.38 & 0.60 & 0.89 \\
\hline $3_{12}-2_{21}$ & 0.21 & 0.67 & 1.6 & 0.40 & 0.67 & 0.84 \\
\hline $3_{21}-3_{12}$ & 0.37 & 1.1 & 2.7 & 0.59 & 1.1 & 1.7 \\
\hline \multicolumn{7}{|c|}{ Para- $\mathrm{H}_{2} \mathrm{O}$ transitions } \\
\hline $1_{11}-0_{00}$ & 0.13 & 1.0 & 0.31 & 0.43 & 1.0 & 1.6 \\
\hline $2_{02}-1_{11}$ & 0.58 & 1.5 & 3.0 & 0.72 & 1.5 & 1.1 \\
\hline $2_{11}-2_{02}$ & 0.34 & 1.0 & 2.0 & 0.41 & 1.0 & 2.7 \\
\hline $2_{20}-2_{11}$ & 0.31 & 1.0 & 2.3 & 0.47 & 1.0 & 1.4 \\
\hline $3_{31}-4_{04}$ & $4.3(-2)$ & 0.15 & 0.58 & 0.18 & 0.15 & $1.3(-3)$ \\
\hline $4_{22}-3_{31}$ & $4.1(-2)$ & 0.13 & 0.48 & 0.13 & 0.13 & $9.6(-2)$ \\
\hline $4_{22}-4_{13}$ & $9.5(-2)$ & 0.26 & 0.74 & 0.18 & 0.26 & $2.3(-3)$ \\
\hline $5_{24}-4_{31}$ & $5.0(-2)$ & 0.15 & 0.57 & 0.16 & 0.15 & $8.7(-2)$ \\
\hline \multirow{2}{*}{\multicolumn{7}{|c|}{$\begin{array}{c}p=2.0 \\
\text { O transitions }\end{array}$}} \\
\hline & & & & & & \\
\hline $1_{10}-1_{01}$ & 0.12 & 0.24 & 0.19 & 0.10 & 0.24 & 0.82 \\
\hline $2_{12}-1_{01}$ & 0.89 & -1.6 & -3.1 & 0.71 & -1.6 & -5.0 \\
\hline $22_{21}-2_{12}$ & 0.13 & 0.14 & 0.15 & 0.13 & 0.14 & -1.4 \\
\hline $3_{12}-3_{03}$ & 0.12 & 0.47 & 1.3 & 0.35 & 0.47 & 0.51 \\
\hline $3_{12}-2_{21}$ & 0.13 & 0.48 & 1.2 & 0.29 & 0.48 & 0.49 \\
\hline $3_{21}-3_{12}$ & 0.20 & 0.87 & 2.1 & 0.45 & 0.87 & 1.2 \\
\hline \multicolumn{7}{|c|}{ Para- $\mathrm{H}_{2} \mathrm{O}$ transitions } \\
\hline $1_{11}-0_{00}$ & 0.30 & $9.4(-2)$ & 0.23 & 0.52 & $9.4(-2)$ & $9.0(-2)$ \\
\hline $2_{02}-1_{11}$ & 0.65 & 1.4 & 3.1 & 0.90 & 1.4 & 1.8 \\
\hline $2_{11}-2_{02}$ & 0.46 & 0.94 & 2.1 & 0.56 & 0.94 & 2.2 \\
\hline $2_{20}-2_{11}$ & 0.32 & 0.78 & 2.1 & 0.54 & 0.78 & 1.3 \\
\hline $3_{31}-4_{04}$ & $-3.7(-3)$ & $-6.7(-3)$ & 0.13 & $7.1(-2)$ & $-6.7(-3)$ & $-1.2(-3)$ \\
\hline $4_{22}-3_{31}$ & $1.0(-2)$ & $5.9(-2)$ & 0.31 & $9.3(-2)$ & $5.9(-2)$ & $2.3(-3)$ \\
\hline $4_{22}-4_{13}$ & $4.7(-2)$ & 0.19 & 0.74 & 0.22 & 0.19 & $8.1(-2)$ \\
\hline $5_{24}-4_{31}$ & $8.3(-3)$ & $6.5(-2)$ & 0.34 & 0.11 & $6.5(-2)$ & $-6.9(-3)$ \\
\hline
\end{tabular}

Columns 2 to 4 show the luminosity variation with a density of $10^{6} \mathrm{~cm}^{-3}$. Columns 5 to 7 show the variation of density with a luminosity of $7 L_{\odot}$.
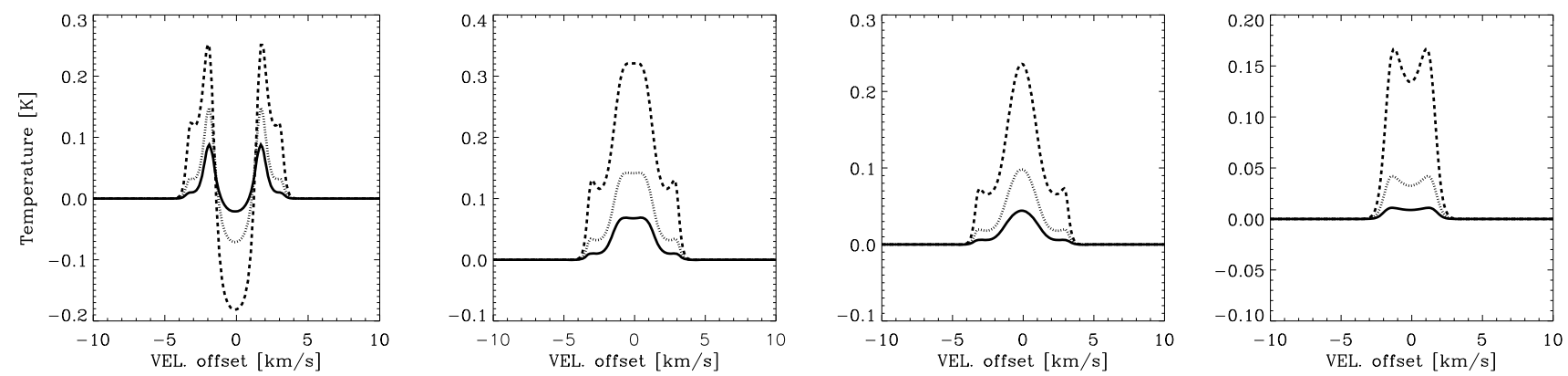

Fig. 5. Examples of the luminosity dependence of line profiles for a model with $p=1.5, X_{0}=10^{-4}, X_{\mathrm{d}}=10^{-8}, n_{0}\left(\mathrm{H}_{2}\right)=10^{6} \mathrm{~cm}^{-3}$. From left to right are shown the $1_{11}-0_{00} 1113 \mathrm{GHz}, 2_{02}-1_{11} 988 \mathrm{GHz}, 2_{11}-2_{02} 752 \mathrm{GHz}$, and $3_{31}-4_{04} 1893 \mathrm{GHz}$ lines. Luminosities are 2 (solid), 7 (dot), and 25 (dash) $L_{\odot}$. These lines reflect the different characteristic line profiles discussed in Sect. 3.4. It can be seen that the line shapes stay the same with increasing luminosity, while the line strengths and depths of the absorption are stronger at higher luminosities.

\subsection{Luminosity}

Figure 5 shows four lines for various luminosities. For all lines, both the line strength and the absorption features are stronger for higher luminosities. For optically thin lines, a best fit can be found with a slope that has a power-law index of $\sim 0.8$, i.e.
$I \propto L^{0.8}$. Figure 7 shows two such power laws for the $22_{20}-2_{11}$ $1228 \mathrm{GHz}$ line that differentiate between the exact conditions and correspond to $0.45 L^{0.8}$ and $0.15 L^{0.8}$. The slope of the power law is determined by the temperature profile, which determines the amount of warm gas present in the dense inner region, and the filling factor of this warm gas within the beam. This filling 

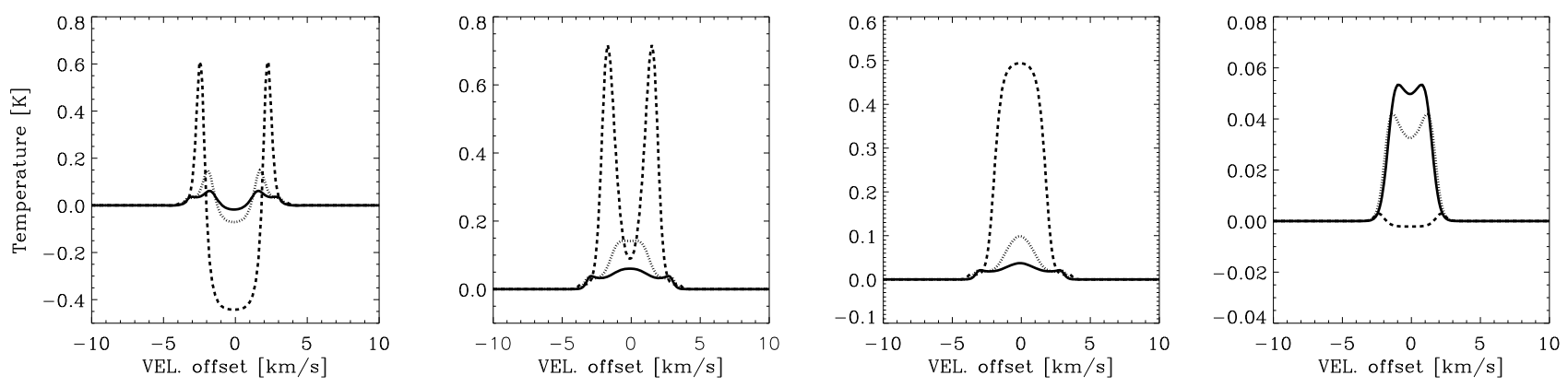

Fig. 6. Examples of the density dependence of line profiles for a model with $p=1.5, X_{0}=10^{-4}, X_{\mathrm{d}}=10^{-8}, L=7 L_{\odot}$. From left to right are shown the $1_{11}-0_{00} 1113 \mathrm{GHz}, 2_{02}-1_{11} 988 \mathrm{GHz}, 2_{11}-2_{02} 752 \mathrm{GHz}$, and $3_{31}-4_{04} 1893 \mathrm{GHz}$ lines. Densities are $4 \times 10^{5}$ (solid), $10^{6}$ (dot), and $5 \times 10^{6}$ (dash) $\mathrm{cm}^{-3}$. For most lines, the higher density, and thus higher water column, increases the emission, although absorption features are also enhanced. For the $3_{31}-4_{04}$ line at $1893 \mathrm{GHz}$, the dust becomes optically thick at lower temperatures (i.e. larger radii) within the envelope for higher densities.

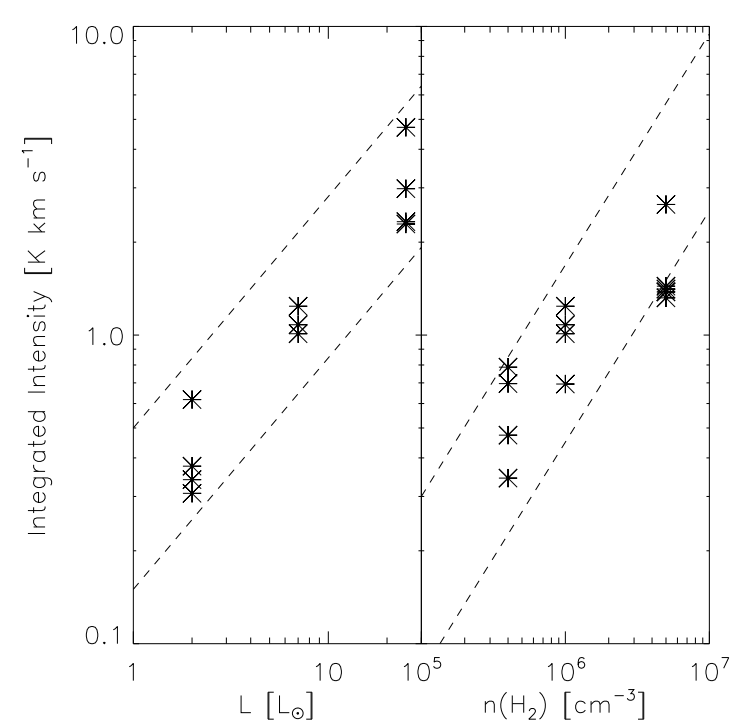

Fig. 7. The modeled integrated intensity of the optically thin $2_{20}-2_{11}$ $1228 \mathrm{GHz}$ line for $X_{0}=10^{-4}$. Intensities are shown for the 3 different values of $X_{\mathrm{d}}$ for a constant density of $10^{6} \mathrm{~cm}^{-3}$ (left) and for a constant luminosity of $7 L_{\odot}(r i g h t)$. Power laws with indices of 0.8 (left) and 0.75 (right) are over-plotted. In the right panel, at $5 \times 10^{6} \mathrm{~cm}^{-3}$, this line becomes optically thick for $X_{\mathrm{d}}=10^{-6}$, with its intensity dropping below the relation.

factor is not readily apparent, since models have different values for $R_{\text {in }}$ and different radii where $T=100 \mathrm{~K}$ is reached depending on luminosity and density profile (see Table 2). The peak temperatures of these optically thin lines naturally follow a similar dependence. Even for lines that display an optically thick line center (e.g., the $331-4_{04}$ line in Fig. 5), the peak temperatures depend on luminosity in a similar way to the completely optically thin lines.

For optically thick lines, a more complex dependency is seen in Fig. 5 (e.g., the $1_{11}-0_{00}$ line). For such self-absorbed lines, the wings (which are optically thin) increase with $L$, while any absorption features also become more prominent. Although the peak temperatures do not follow the proposed power law of the optically thin lines, they will become easier to detect for more luminous sources.

\subsection{Density}

Figure 6 shows four lines for a range of densities, varying by more than an order of magnitude, corresponding to envelope masses ranging from 0.15 to $1.93 M_{\odot}$. The density at $1000 \mathrm{AU}$ has a strong influence on the water line emission. For optically thin lines, models with a constant $p$ and constant luminosity show that a power law with an index of 0.75 accurately describes the dependence of line strengths on $n_{0}\left(\mathrm{H}_{2}\right)$ (see Fig. 7). For optically thick lines, the line will decrease in strength at higher densities, since the emission from the warm inner part is veiled behind a larger, cold water column.

A higher density can also result in the dust becoming optically thick. This has an effect on the higher excited lines at high frequencies. For example, the optically thin $3_{31}-4_{04} 1893 \mathrm{GHz}$ line (see Fig. 6, right) decreases by nearly two orders of magnitude in strength when the density increases from $0.4 \times 10^{6}$ to $5 \times 10^{6} \mathrm{~cm}^{-3}$. The dust is optically thin in the low-density models and optically thick at high density. Since the emission of this higher excitation line originates in the warm inner region, optically thick dust can obscure over $99 \%$ of the emission of this high frequency line (see Sect. 5).

\subsection{Density profile index}

The steepness of the power law used to define the density distribution, $p$, also influences water line emission. Table 6 shows that lines differ subtly between $p=2$ and $p=1.5$. The line intensities of models with $p=2$ are lower by a factor ranging from 0.5 to 3 than those for models with $p=1.5$. Absorption features also differ in their depths for similar models with only $p$ varied. This is caused by differing temperature profiles within the Herschel beams. Assuming equal $n_{0}\left(\mathrm{H}_{2}\right)$ at $1000 \mathrm{AU}$, the warm $(T>100 \mathrm{~K})$ region is significantly smaller for a model with $p=2$ than for $p=1.5$. Although local densities are higher by almost an order of magnitude, the beam dilution is too severe to overcome such small regions.

\section{4. $\mathrm{H}_{2}^{18} \mathrm{O}$}

The models were also calculated for the isotopologue $\mathrm{H}_{2}^{18} \mathrm{O}$. Similar to the L 483 models, transitions connected with the ground state lines are optically thick for all models. $\mathrm{H}_{2}^{18} \mathrm{O}$ excited lines with $J_{\text {up }}=2$ and 3 do have optically thick centers for some combinations of abundance, luminosities and/or densities, but are optically thin at densities below $10^{6} \mathrm{~cm}^{-3}$. Higher 
excitation $\mathrm{H}_{2}^{18} \mathrm{O}$ lines are always optically thin. The best-fit power law slopes for the optically thin $\mathrm{H}_{2}^{18} \mathrm{O}$ lines are the same as those for $\mathrm{H}_{2}^{16} \mathrm{O}$. Of course, these conclusions are limited by the optical depth of dust at the frequency of the line, since $\mathrm{H}_{2}^{18} \mathrm{O}$ lines at high frequencies are affected by dust. The precise frequency depends on the combination of luminosity and the density profile (Sect. 5). Results for $\mathrm{H}_{2}^{18} \mathrm{O}$ can be found in the online appendix.

\subsection{Line profiles}

The $\mathrm{H}_{2} \mathrm{O}$ line profiles shown in Figs. 5 and 6 all contain optically thin and optically thick parts as discussed in Sect. 3.4. Since the line wings of most transitions are optically thin, a "bump" is present in these wings at the velocity for which that particular line becomes optically thick. This velocity depends solely on the total column of water at this velocity and not on the luminosity. The height of this bump is determined by both the luminosity and the density profile. The line center changes according to the actual optical depth of both the dust and the water itself. Line centers can (i) be effectively thin and show a Gaussian profile, (ii) be dominated by a small region and be flat-topped, (iii) show a self-absorption feature determined by the optical depth of the line alone; (iv) have absorption into the dust continuum.

\subsection{Model limitations}

Our spherically symmetric envelope models have several limitations, which can affect the interpretation of observed line strengths.

- The presence of a large velocity gradient, such as seen in infall and rotation of envelopes around high-mass stars, can significantly broaden the water line and simultaneously make it less optically thick. The velocity field in these models was taken to be small, with an average infall velocity of $4 \mathrm{~km} \mathrm{~s}^{-1}$ at $R_{\text {in }}$. For low-mass sources, such an infall velocity combined with a turbulent width of $1 \mathrm{~km} \mathrm{~s}^{-1}$ has been shown to accurately reproduce observed emission line profiles (Jørgensen 2004).

- Outflow contributions. No outflow contribution has been taken into account in our models. It is assumed that emission from outflow material can be identified with sufficient spectral resolution in observational data with Herschel-HIFI. However, material heated by outflow shocks in high-density regions will not necessarily be accelerated to high velocities. This material can add significantly to the emission of water lines, but requires a detailed shock model to quantify it (e.g., Kaufman \& Neufeld 1996). In addition, outflow cavities allow emission from the high-density regions of the inner envelope to escape along paths with much lower density than encountered in the cold outer envelope.

- Large extended molecular cloud or fore-ground cloud contributions can both emit rotational water lines or absorb radiation coming from the protostellar envelope. This is especially likely for the three transitions connected to the ground-states of ortho- and para-water. For example, for a cloud column density of $10^{22} \mathrm{~cm}^{-2}$ and an assumed water abundance of $10^{-8}$ with a gas density of $10^{5} \mathrm{~cm}^{-3}$, the ground state ortho$\mathrm{H}_{2} \mathrm{O} 557 \mathrm{GHz}$ line will have an intensity of $50 \mathrm{mK}$ if the emission fills the beam. The optical depth of this line is large enough, $\tau \approx 20$, to absorb radiation coming from behind this cloud. Results from SWAS and ODIN (See Sect. 6.2)
Table 7. Integrated intensity and peak brightness of lines with and without dust for a model with $p=1.5, L=7 L_{\mathrm{bol}}$ and $n_{\mathrm{H}_{2}}=1 \times 10^{6} \mathrm{~cm}^{-3}$.

\begin{tabular}{lllll}
\hline \hline Transition & \multicolumn{2}{c}{ Dust } & \multicolumn{2}{c}{ No dust } \\
& Intensity & $T_{\text {peak }}$ & Intensity & $T_{\text {peak }}$ \\
& {$\left[\mathrm{K} \mathrm{km} \mathrm{s}^{-1}\right]$} & {$[\mathrm{K}]$} & {$\left[\mathrm{K} \mathrm{km} \mathrm{s}^{-1}\right]$} & {$[\mathrm{K}]$} \\
\hline $1_{10}-1_{01}$ & 0.95 & 0.47 & 1.41 & 0.65 \\
$2_{12}-1_{01}$ & $-2.6(-2)$ & 0.26 & 0.16 & 0.39 \\
$3_{12}-3_{03}$ & 0.67 & 0.18 & 0.39 & 0.06 \\
\hline
\end{tabular}

indeed do not show the deep absorption in the $557 \mathrm{GHz}$ line, seen in Figs. 3-6. It is likely that extended cold cloud material dominates close $\left(<0.5 \mathrm{~km} \mathrm{~s}^{-1}\right)$ to the line center in these data due to the much larger observing beams than Herschel. For excited lines, emission and optical depth in a foreground cloud are negligible. At high ( $>1 \mathrm{THz})$ frequencies, the dust within such cloud complexes can, however, absorb the emission from excited water.

- A large massive gas disk can contribute to the emission of water. However, most gas within disks is at low $(T \sim 30 \mathrm{~K})$ temperatures. Most of the water will be frozen out, except in the inner few $\mathrm{AU}$ and in the warm surface layers. In addition, high dust columns can completely obscure frequencies above $1 \mathrm{THz}$. Water emission from disks is thus expected to be orders of magnitude lower for lines at all frequencies, except for the transitions connected to the ground state below $1 \mathrm{THz}$. However, the small angular size of disks causes much of the emission to be diluted to much lower values (a few $\mathrm{mK}$ ) within the Herschel beam than commonly found for envelopes (a few $100 \mathrm{mK}$ to a few K).

\section{Effects on $\mathrm{H}_{2} \mathrm{O}$ excitation and line formation: dust and micro-turbulence}

\subsection{Dust}

The presence of dust in protostellar envelopes influences water emission lines significantly in two ways, as can be seen in Table 7. First, the dust can become optically thick, preventing water emission from deep in the envelope to escape. Second, the far-infrared radiation can pump the water lines. Dust becomes optically thick at specific columns, depending on the value of the opacity, $\kappa$, at a given frequency (see Fig. $5 \mathrm{c}$ in Ossenkopf $\&$ Henning 1994, for the precise relation). Since $\kappa_{v} \propto v^{\beta}$, with the dust opacity index $\beta$ typically 1.5 , this is especially likely at higher frequencies. Column 4 of Table 8 shows the total gas and dust column densities needed to reach a dust optical depth equal to 1 for each line.

Figure 8 illustrates this effect in a cartoon for two different envelope models. Two models, one with low density (low $M_{\mathrm{env}} \approx$ $0.15 M_{\odot}$ ) and one with high density (high $M_{\mathrm{env}} \approx 1.93 M_{\odot}$ ), are compared for two ground-state lines. The dust at $557 \mathrm{GHz}$ only becomes optically thick for the more massive envelope due to very high column densities, while the low-density model remains optically thin throughout. The $\tau=1$ surfaces correspond to temperatures well over $100 \mathrm{~K}$. Since the $557 \mathrm{GHz}$ water line is optically thick at lower columns, the dust will have no effect on this line, except for weakening the line due to dust absorption. Even the $\mathrm{H}_{2}^{18} \mathrm{O}$ column is large enough to prevent the dust opacity from influencing the $548 \mathrm{GHz}$ line. In contrast, the dust at $1669 \mathrm{GHz}$ becomes optically thick at a much smaller column, which corresponds to a radius and temperature much farther out into the envelope. The actual radius depends on the 
Table 8. The dust properties at selected water line frequencies for four typical models. The column density $N_{\mathrm{H}_{2}}$ required to produce $\tau=1$ for the dust at each frequency is given with the corresponding radii and temperatures.

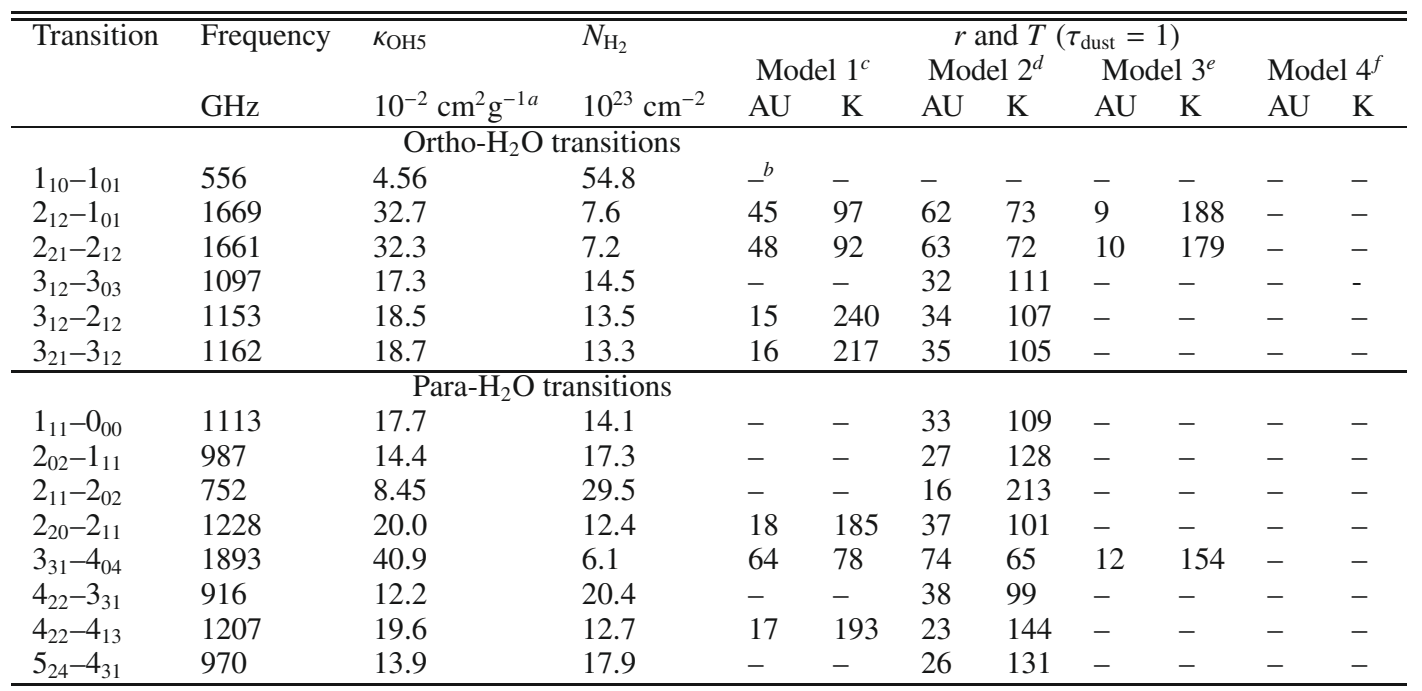

${ }^{a}$ Total gas and dust; ${ }^{b}$-indicates that the dust is optically thin throughout the entire envelope; ${ }^{c}$ see text, $L=7 L_{\odot}, p=1.5, n_{0}=5 \times 10^{6} \mathrm{~cm}^{-3}$; ${ }^{d}$ see text, $L=7 L_{\odot}, p=2, n_{0}=5 \times 10^{6} \mathrm{~cm}^{-3}$; ${ }^{e}$ see text, $L=2 L_{\odot}, p=2, n_{0}=4 \times 10^{5} \mathrm{~cm}^{-3} ;{ }^{f}$ see text, $L=25 L_{\odot}, p=1.5, n_{0}=1 \times 10^{6} \mathrm{~cm}^{-3}$.

model in question. In the low-density model, the dust only turns optically thick at radii close to the $100 \mathrm{~K}$ water evaporation radius, while dust in the high-density model already obscures the line in the outer regions of the envelope. Optically thin lines at this frequency will not be able to probe the warm inner region, since the optically thick dust effectively absorbs all water line emission.

Figure 9 shows example spectra of the $1_{10}-1_{01}$ line at $557 \mathrm{GHz}, 2_{12}-1_{01}$ line at $1669 \mathrm{GHz}$ and the $3_{12}-3_{03}$ line at $1097 \mathrm{GHz}$ for a model with $L=7 L_{\odot}, n_{\mathrm{H}_{2}}=1 \times 10^{6} \mathrm{~cm}^{-3}$, $p=1.5, X_{0}=10^{-4}$, and $X_{\mathrm{d}}=10^{-7}$, with and without dust. The spectra with dust are significantly weaker by up to $50 \%$, even for optically thin dust at $557 \mathrm{GHz}$, since dust within the line of sight absorbs the water line photons. Lines that are optically thin at a frequency where optically thick dust exists are weaker by factors up to a few or more if dust is present, due to less water being "visible". Optically thin lines can be pumped by far-infrared dust radiation coming from the region at radii larger than the $\tau=1$ surface of the dust at the frequency of the line.

For each envelope model, the column density of dust depends on a combination of luminosity $L$ (through the location of $R_{\text {in }}$, Table 2) and envelope structure parameters $n_{0}$ and $p$. If the $\tau=1$ surface is approximated as a hard surface where all water photons are absorbed, a typical temperature can be extracted for each combination of $L, n_{0}$, and $p$. This temperature is the upper limit to which water lines can probe for each model before dust becomes optically thick. Table 8 presents the $\tau=1$ temperatures for four astronomically distinct models, based on the results from Table 6 of Jørgensen et al. (2002). Model 1, with $L=7 L_{\odot}, p=1.5$, and $n_{0}=5 \times 10^{6} \mathrm{~cm}^{-3}$, corresponds to a very young Class $0 \mathrm{YSO}$, which is dominated by a large, cold, and massive protostellar envelope. A good example is the well-studied NGC 1333 IRAS 4B source in the Perseus molecular cloud. Lines with a frequency above $1600 \mathrm{GHz}$ are unable to probe the region with $T>100 \mathrm{~K}$. Dust is optically thin throughout the envelope for frequencies lower than a $1000 \mathrm{GHz}$. Model 2, with $L=7 L_{\odot}, p=2$ and $n_{0}=5 \times 10^{6} \mathrm{~cm}^{-3}$, has similar parameters but its mass is more centrally condensed. Due to the very high column densities, dust becomes optically thick at all frequencies above $700 \mathrm{GHz}$, making it even more difficult to probe the central warm region than in Model 1. Highly excited water lines are effectively shielded from observers within this envelope at all frequencies. The third model, $L=2 L_{\odot}, p=2$, $n_{0}=4 \times 10^{5} \mathrm{~cm}^{-3}$, corresponds to a more evolved source with a low luminosity and a small envelope with most of the mass concentrated in the center due to a steep density profile. The relative amount of warm gas in this envelope is significantly higher. Such a model would correspond to most Class I sources, e.g., TMC-1A. The last model included has $L=25 L_{\odot}, p=1.5$, and $n_{0}=1 \times 10^{6} \mathrm{~cm}^{-3}$ which corresponds to a higher luminosity source, but with a low total mass. The mass of the envelope is distributed over larger radii and the luminosity is higher. For these last two models, dust will not hide the inner parts of the envelope at all. Only the very warm region $(T>150 \mathrm{~K})$ is veiled near the inner radius of Model 3.

\subsection{Micro-turbulence}

The structure of micro-turbulence, defined in our models as a velocity dispersion, during the embedded phases of star formation is not well known, and theoretical models do not yet agree on a correct treatment. A good review of the theoretical considerations and problems is given in Ward-Thompson \& Buckley (2001, their Sect. 3.2). Jørgensen (2004) concludes that the inclusion of micro-turbulence as a velocity dispersion gives a more accurate reproduction of line intensities and profiles as opposed to the inclusion of infall (Jørgensen et al. 2005). Ward-Thompson \& Buckley (2001, Sect. 4.6) test different values of micro-turbulence, as well as different power-law values for the variation of micro-turbulence with radius. This results in a large variation in the molecular line profiles of $\mathrm{CS}$ and $\mathrm{HCO}^{+}$. Similarly, Fig. 10 shows that the choice of the velocity dispersion can have a strong effect on all water lines, both optically thick and thin. All three lines $(557,1669$, and $1097 \mathrm{GHz})$ show large differences in both line profile and in total integrated emission up to a factor of 3 , when the velocity dispersion is varied between $0.83(b=0.5), 1.67(b=1)$ and $3.34(b=2) \mathrm{km} \mathrm{s}^{-1}$. Absorption both into the continuum and in the line are broader 


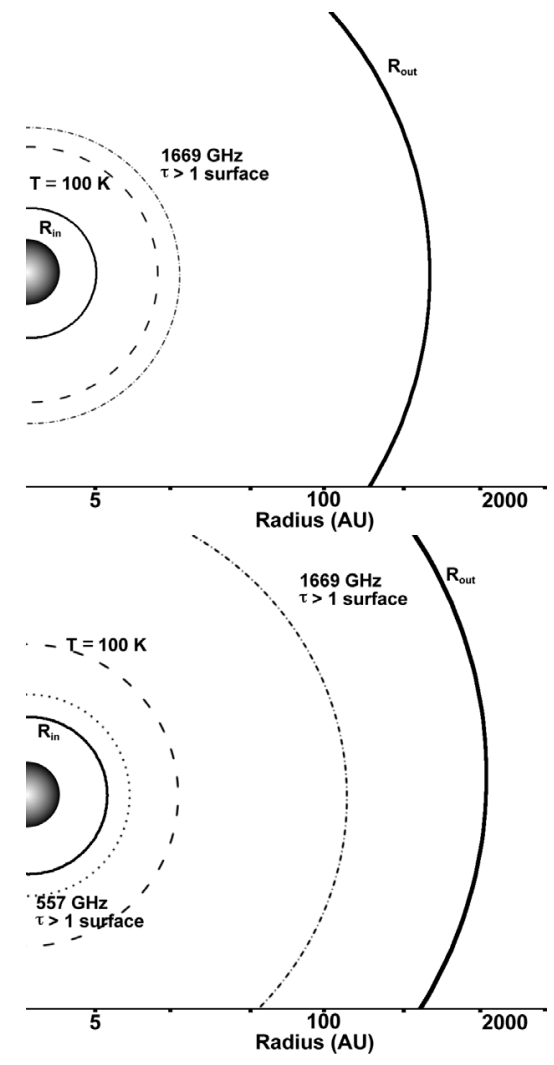

Fig. 8. Cartoon of protostellar envelope models. For two different models, the $\tau=1$ surfaces at 557 and $1669 \mathrm{GHz}$ for the dust are shown. A model with low-density $\left(n_{0}\left(\mathrm{H}_{2}\right)=4 \times 10^{5} \mathrm{~cm}^{-3}, M=0.15 M_{\odot}\right)$ is shown in the top panel; here the $557 \mathrm{GHz}$ line contour is absent, since dust is optically thin throughout the envelope at this frequency. A model with a high density $\left(n_{0}\left(\mathrm{H}_{2}\right)=5 \times 10^{6} \mathrm{~cm}^{-3}, M=1.93 M_{\odot}\right)$ is shown in the bottom panel. The long-dashed line indicates the $T=100 \mathrm{~K}$ contour. In both models, the $1669 \mathrm{GHz}$ line is unable to probe the inner warm region, regardless of its own optical depth, since the dust $\tau=1$ surface lies far outside the $T=100 \mathrm{~K}$ radius.

for higher values of micro-turbulence, while peak separation increases with increased velocity dispersion. Optically thin lines and line wings broaden if $b$ is larger. Such effects are similar to those found by Ward-Thompson \& Buckley (2001).

For molecular lines in low-mass protostellar envelopes, Ward-Thompson \& Buckley (2001) and Jørgensen et al. (2005) both adopt a constant value for the micro-turbulence with radius. In our case, a velocity dispersion, $\Delta V \sim 1.67 \mathrm{~km} \mathrm{~s}^{-1}$ $\left(b=1 \mathrm{~km} \mathrm{~s}^{-1}\right)$, has been chosen to best reproduce line emission for a wide variety of molecules in sources such as L483, including $\mathrm{CO}, \mathrm{CS}$, but also complex molecules such as $\mathrm{H}_{2} \mathrm{CO}$ and $\mathrm{CH}_{3} \mathrm{OH}$, tracing both the warm and cold regions. We recommend that for the modeling of water lines from specific sources, the velocity dispersion for the cloud in question be adopted as derived from other molecular lines.

\section{Observational studies}

The conclusions presented in previous paragraphs can be compared to current observational studies. However, due to the approximations used within the models and the unique combinations of parameters for individual sources, it is recommended that these models be used only as a starting point for interpreting water emission of protostellar envelopes from specific sources. As discussed in Sect. 4.6, there are several factors that will complicate any analysis of observed water lines. To compare the envelope models presented above with any individual observation other than those from Herschel, the line intensities have to be corrected for the actual beam size of the telescope.

\subsection{ISO-LWS}

Water has been detected in several low-mass YSOs (both Class 0 and Class I) with ISO-LWS such as NGC 1333 IRAS 2/4, IRAS 16293-2422, Elias 29, L 1448, L 483, and SSV 13 (e.g., Liseau et al. 1996; Ceccarelli et al. 1999; Nisini et al. 1999; Giannini et al. 2001; Maret et al. 2002). Up to 14 water lines were detected, ranging from $180.5 \mu \mathrm{m}$ to $67.3 \mu \mathrm{m}$. In most sources the total number of lines is significantly lower, but sufficient for proving the existence of water. For example, only a single line was detected for the Class I source Elias 29 (Ceccarelli et al. 1999).

As an illustration, we consider the case of NGC 1333 IRAS4A/B, for which Maret et al. (2002) report the emission of 14 water lines, together with high-J CO, OH, [O I], and [C II]. A model was put forward to explain the origin of these FIR lines using a collapsing envelope model without an outflow. Based on the model developed by Ceccarelli et al. (1996), the envelope and stellar parameters were derived from the best fit model to the 14 water lines. The water abundances in the envelope were found to include a jump at $100 \mathrm{~K}$ with a best fit of $X_{0}=5 \times 10^{-6}$ and $X_{\mathrm{d}}=5 \times 10^{-7}$. A lower inner abundance is excluded by their model results, but a higher $X_{0}$ abundance of the order of $5 \times 10^{-5}$ cannot be excluded.

The physical parameters derived by Jørgensen et al. (2002) and abundances proposed by Maret et al. (2002) correspond best with our model to $n_{0}=10^{6} \mathrm{~cm}^{-3}, L=7 L_{\odot}$, and $p=1.5$ with abundances $10^{-6}$ for $X_{0}$ and $10^{-6} / 10^{-7}$ for $X_{\mathrm{d}}$. However, our predicted line strengths differ from the observed and predicted line strengths in Maret et al. (2002), especially for low excitation lines. Our $2_{21}-2_{12}$ and $2_{12}-1_{01}$ predictions are $0.510^{-20}$ and $1.1 \times 10^{-20} \mathrm{~W} \mathrm{~cm}^{-2}$ for the $10^{-6} / 10^{-7}$ abundance combination within the ISO-LWS beam, significantly lower than the observed values of 11 and $27 \times 10^{-20} \mathrm{~W} \mathrm{~cm}^{-2}$. Although an inner abundance of $10^{-4}$ produces brighter lines for the presented models, these lines are still an order of magnitude lower than the observed fluxes in Maret et al. (2002). The most likely explanation is the different treatment of dust between the two models, which lowers the fluxes of the high-frequency lines in our case. However, this also suggests that our models can only explain the ISO-LWS fluxes if there are cavities in the envelopes through which the photons can escape. Recent results with the Spitzer Space Telescope show numerous spectrally unresolved water lines from 20 to $30 \mu \mathrm{m}$, which suggest that NGC 1333 IRAS $4 \mathrm{~B}$ is indeed viewed almost face-on, allowing the ro-vibrational lines to escape the protostellar system unhindered along the outflow axis (Watson et al. 2007). This can also help the longer wavelength lines coming from the inner envelope. Another possibility is that significant contributions come from large-scale outflows. This is confirmed by SWAS (see Sect. 6.2).

\subsection{SWAS}

SWAS $^{5}$ observed only the ground-state transition of $\mathrm{o}-\mathrm{H}_{2} \mathrm{O}$ at $557 \mathrm{GHz}$ and $\mathrm{o}-\mathrm{H}_{2}^{18} \mathrm{O}$ at $548 \mathrm{GHz}$. Although the beam of SWAS $\left(3.3^{\prime} \times 4.5^{\prime}\right)$ is significantly larger than for ISO-LWS, the

\footnotetext{
5 See http://cfa-www. harvard. edu/swas/ for more information.
} 

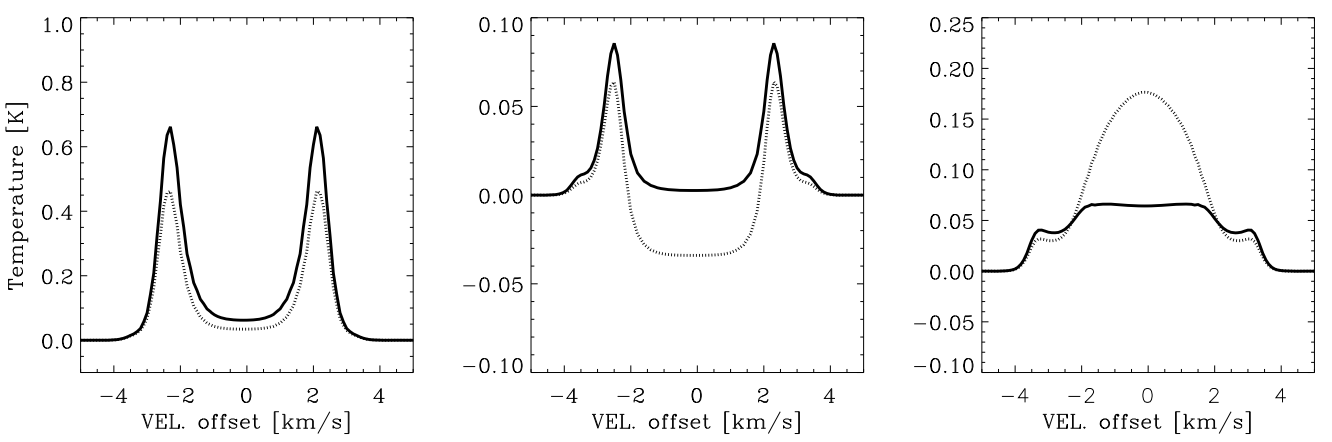

Fig. 9. Example spectra of the $1_{10}-1_{01} 557 \mathrm{GHz}$ line (left), $2_{12}-1_{01} 1669 \mathrm{GHz}$ line (middle) and $3_{12}-3_{03} 1097 \mathrm{GHz}$ line (right) for a model with $p=1.5, L=7 L_{\odot}$ and $n_{\mathrm{H}_{2}}=1 \times 10^{6} \mathrm{~cm}^{-3}$. The solid lines represent the spectra with no dust within the envelope, while the dotted line shows the same line with dust included in the model. The influence of dust can be clearly seen. The peaks of the optically thick lines are reduced in strength, the depth of the absorption changes, and the optically thin lines are significantly pumped by far-infrared radiation.
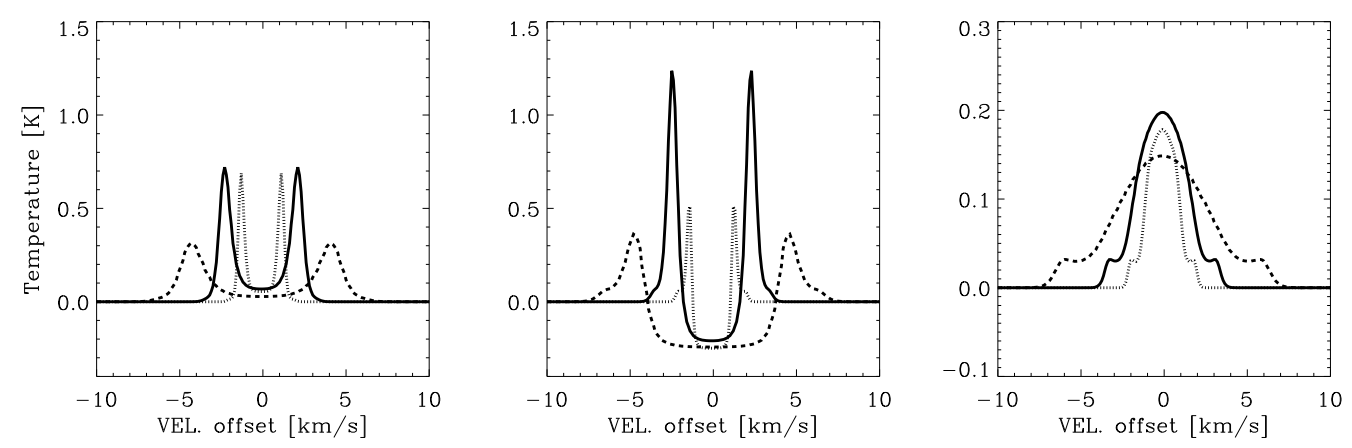

Fig. 10. Example spectra of the $1_{10}-1_{01} 557 \mathrm{GHz}$ line (left), $2_{12}-1_{01} 1669 \mathrm{GHz}$ line (middle), and $3_{12}-3_{03} 1097 \mathrm{GHz}$ line (right) for a model with $p=1.5, L=7 L_{\odot}$, and $n_{\mathrm{H}_{2}}=1 \times 10^{6} \mathrm{~cm}^{-3}$. The dotted line is for a model with $\Delta V=0.83 \mathrm{~km} \mathrm{~s}^{-1}$. The solid line for $\Delta V=1.67 \mathrm{~km} \mathrm{~s}{ }^{-1}$, and the dashed line for $\Delta V=3.34 \mathrm{~km} \mathrm{~s}^{-1}$. The large effect of micro-turbulence for all lines can be seen clearly.

increased spectral resolution of better than $1 \mathrm{~km} \mathrm{~s}^{-1}$ spectrally resolves most lines in starforming regions.

SWAS observed the NGC 1333 IRAS4 region (Neufeld et al. 2000; Bergin et al. 2003) where, contrary to the analysis of Maret et al. (2002), they observed a wide $\left(24.4 \mathrm{~km} \mathrm{~s}^{-1}\right)$ and bright $\left(\int T_{A}^{*} \mathrm{~d} V=0.5-1.9 \mathrm{~K} \mathrm{~km} \mathrm{~s}^{-1}\right)$ line. It was concluded that these lines originate within the outflow of one or more sources inside the NGC 1333 region. The model proposed by Maret et al. (2002) would have produced a narrow $\left(<4 \mathrm{~km} \mathrm{~s}^{-1}\right)$ and weak $\left(0.02-0.04 \mathrm{~K} \mathrm{~km} \mathrm{~s}^{-1}\right) 557 \mathrm{GHz}$ line for each source contained within the beam. Our grid gives a typical integrated intensity within a SWAS beam of $\int T_{\mathrm{MB}} \mathrm{d} V=0.5 \mathrm{~K} \mathrm{~km} \mathrm{~s}^{-1}$ for a $X_{0} / X_{\mathrm{d}}$ combination of $10^{-4} / 10^{-6}$ with a typical line width of $6 \mathrm{~km} \mathrm{~s}^{-1}$.

Ashby et al. (2000) report on SWAS data for B335, an isolated Class 0 source with a $3 \sigma$ upper limit of $<0.06 \mathrm{~K} \mathrm{~km} \mathrm{~s}^{-1}$. Evans et al. (2005) used a wide variety of molecular emission lines to model the chemistry and physical structure of this source. The low luminosity $\left(4.5 L_{\odot}\right)$ and low density $(4 \times$ $10^{5} \mathrm{~cm}^{-3}$ ) found by Evans et al. (2005) indicate that this class 0 source would be represented best by a model with $p=1.5$, $L=2 L_{\odot}$ and $n_{0}\left(\mathrm{H}_{2}\right)=4 \times 10^{5} \mathrm{~cm}^{-3}$. Our predicted $557 \mathrm{GHz}$ line strength corrected for the much larger SWAS beam is 0.02 $0.06 \mathrm{~K} \mathrm{~km} \mathrm{~s}^{-1}$ for outer abundances from $10^{-8}$ to $10^{-6}$, just below the upper limits from SWAS. The only model that would have been detectable with SWAS has a constant abundance of $10^{-6}$. The high jump abundance model with $X_{0}=10^{-4}$ and $X_{\mathrm{d}}=$ $10^{-6}$ cannot be ruled out, since most of the line is self-absorbed.
A similar conclusion holds for most other Class 0 sources detected with SWAS.

Using the SWAS archive ${ }^{6}$, upper limits were found on several Class I sources, including RCrA IRS 5 and 7. Only upper limits of $20-50 \mathrm{mK}$ in peak temperature could be derived. These observed limits are higher by up to an order of magnitude than the predictions presented in Sect. 4 for models that would fit the physical parameters of Class I sources. The cold envelope material is constrained to $10^{\prime \prime}-30^{\prime \prime}$, a small region within the SWAS beam. Thus, our models are consistent with the scarce observational data on Class I sources.

In summary, both the ISO and SWAS data stress the need for Herschel Space Observatory data to properly constrain the origin of the observed $\mathrm{H}_{2} \mathrm{O}$ emission and distinguish large-scale and outflow contributions from the emission originating in the quiescent gas of the protostellar envelope.

\section{Summary and conclusions}

This paper presents several models that examine the excitation of water and its oxygen-18 isotopologue in envelopes around low-mass protostars, with parameters covering the entire range of luminosity and envelope characteristics of observed Class 0 and I sources. A model for the Class 0 source L 483 was used to illustrate the dependence of rotational water lines on the water

\footnotetext{
${ }^{6}$ See http://lambda.gsfc.nasa.gov/product/swas/ for more information.
} 
abundances in the inner $(T>100 \mathrm{~K})$ region $X_{0}$ and outer $(T<$ $100 \mathrm{~K}$ ) region $X_{\mathrm{d}}$. In turn, a broader grid of models was explored for the full range of parameters and likely abundances. The main conclusions follow.

- The strengths and line shapes of all rotational water lines are sensitive to the physical parameters of the envelope. Thus, an accurate physical model, including a well-constrained density profile, is a necessary pre-requisite to modeling the water emission and derive abundances.

- Ground-state lines from both $\mathrm{H}_{2}^{16} \mathrm{O}$ and $\mathrm{H}_{2}^{18} \mathrm{O}$ become optically thick at low water columns. Even though water abundances are expected to be very low in the outer envelope $\left(10^{-8}\right.$ with respect to $\left.\mathrm{H}_{2}\right)$, the ground-state lines are still dominated by emission from the cold $(T<30 \mathrm{~K})$ outer $(r>1000 \mathrm{AU})$ envelope. Ground-state line profiles are characterized by a double-peak feature with an absorption around narrow, optically thin emission line wings. The total integrated intensity cannot be used to trace the water abundance. Spectrally resolving the ground state lines is essential if these lines are to be used as probes for the water abundance.

- Higher excitation lines $\left(E_{\text {up }}>150 \mathrm{~K}\right)$ are usually dominated by emission coming from the warm inner region. At very high excitation $\left(E_{\text {up }}>200-250 \mathrm{~K}\right)$, the lines are optically thin. Although these lines are weak $\left(<0.4 \mathrm{~K} \mathrm{~km} \mathrm{~s}^{-1}\right)$, they provide valuable information about the characteristics of the warm gas present in the inner regions of the protostellar envelopes. Power laws with an index of 0.8 and 0.75 were found to accurately represent the dependence of optically thin lines on luminosity and density.

- $\mathrm{H}_{2}^{18} \mathrm{O}$ is a reliable alternative tracer of the abundances in both the warm $(T>100 \mathrm{~K})$ and cold $(T<100 \mathrm{~K})$ regions of the envelope. However, such lines are much weaker with predicted peak temperatures of only a few tens of $\mathrm{mK}$, requiring very long integration times.

- The presence of dust strongly influences the emission of most water lines. The optical depth of dust, which depends on the luminosity (due to varying $R_{\text {in }}$ in our models) and density profile parameters, can prevent optically thin water lines from escaping the inner envelope, resulting in orders of magnitude reduction of the water fluxes. In addition, even optically thin dust absorbs up to $50 \%$ of the total line emission. Furthermore, the far-infrared continuum can pump optically thin water lines.

- The value of the micro-turbulent velocity dispersion and the structure of any systematic velocity field can significantly influence the line profiles and intensities of all water lines. The adoption of a micro-turbulent width that is constant with radius, as inferred from line profile fits of other molecules such as $\mathrm{CS}$ and $\mathrm{HCO}^{+}$, is expected to produce the best-fitting $\mathrm{H}_{2} \mathrm{O}$ line profiles, but the actual values need to be determined on a source-by-source basis.

To conclude, although water is a complex molecule to model, total intenstities and line profiles of rotational water transitions can be used to constrain essential information about the protostellar envelope. The increased sensitivity and resolution, both spectrally and spatially available with HIFI and PACS on Herschel compared with previous missions, will provide the technology to observe water lines covering a wide range of excitations for the water molecule. The increased spectral resolution will be essential for locating the origin of warm gaseous water and understanding the structure of low-mass protostellar envelopes. Abundances in both the cold outer region and the warm inner region are best probed by a combination of lines and must include detailed line profiles.

Acknowledgements. T.v.K. would like to thank Dieter Poelman, Floris van der Tak, and Antonio Crapsi for valuable discussion concerning YSO envelope modeling in general and the use of RATRAN. T.v.K. and astrochemistry at Leiden Observatory are supported by a Spinoza grant and by grant 614.041.004 from the Netherlands Organization for Scientific Research (NWO).

\section{References}

Adams, F. C., Lada, C. J., \& Shu, F. H. 1987, ApJ, 312, 788

André, P., Ward-Thompson, D., \& Barsony, M. 1993, ApJ, 406, 122

André, P., Ward-Thompson, D., \& Barsony, M. 2000, Protostars and Planets IV, 59

Ashby, M. L. N., Bergin, E. A., Plume, R., et al. 2000, ApJ, 539, L119

Bergin, E. A., \& Snell, R. L. 2002, ApJ, 581, L105

Bergin, E. A., \& Melnick, G. J. 2005, in Astrochemistry: Recent Successes and Current Challenges, ed. D. C. Lis, G. A. Blake, \& E. Herbst, IAU Symp., 231, 309

Bergin, E. A., Kaufman, M. J., Melnick, G. J., Snell, R. L., \& Howe, J. E. 2003, ApJ, 582, 830

Boogert, A. C. A., Hogerheijde, M. R., Ceccarelli, C., et al. 2002, ApJ, 570, 708

Boonman, A. M. S., Doty, S. D., van Dishoeck, E. F., et al. 2003, A\&A, 406, 937

Butner, H. M., Charnley, S. B., Ceccarelli, C., et al. 2007, ApJ, 659, L137

Ceccarelli, C., Hollenbach, D. J., \& Tielens, A. G. G. M. 1996, ApJ, 471, 400

Ceccarelli, C., Caux, E., Loinard, L., et al. 1999, A\&A, 342, L21

Ceccarelli, C., Castets, A., Caux, E., et al. 2000, A\&A, 355, 1129

Ceccarelli, C., Baudry, A., Caux, E., et al. 2001, in The Promise of the Herschel Space Observatory, ed. G. L. Pilbratt, J. Cernicharo, A. M. Heras, T. Prusti, \& R. Harris, ESA SP-460, 219

Cernicharo, J., \& Crovisier, J. 2005, Space Sci. Rev., 119, 29

Cernicharo, J., Thum, C., Hein, H., et al. 1990, A\&A, 231, L15

Cernicharo, J., Bachiller, R., \& Gonzalez-Alfonso, E. 1996, A\&A, 305, L5

Charnley, S. B., Tielens, A. G. G. M., \& Millar, T. J. 1992, ApJ, 399, L71

Charnley, S. B., Rodgers, S. D., \& Ehrenfreund, P. 2001, A\&A, 378, 1024

de Graauw, T., \& Helmich, F. P. 2001, in The Promise of the Herschel Space Observatory, ed. G. L. Pilbratt, J. Cernicharo, A. M. Heras, T. Prusti, \& R. Harris, ESA SP-460, 45

Doty, S. D. 2000, ApJ, 535, 907

Doty, S. D., \& Leung, C. M. 1994, ApJ, 424, 729

Doty, S. D., \& Neufeld, D. A. 1997, ApJ, 489, 122

Dubernet, M.-L., \& Grosjean, A. 2002, A\&A, 390, 793

Dubernet, M.-L., Daniel, F., Grosjean, A., et al. 2006, A\&A, 460, 323

Elitzur, M., Hollenbach, D. J., \& McKee, C. F. 1989, ApJ, 346, 983

Evans, II, N. J., Lee, J.-E., Rawlings, J. M. C., \& Choi, M. 2005, ApJ, 626, 919

Faure, A., Crimier, N., Ceccarelli, C., et al. 2007, A\&A, 472, 1029

Felli, M., Brand, J., Cesaroni, R., et al. 2007, A\&A, 476, 373

Fraser, H. J., Collings, M. P., McCoustra, M. R. S., \& Williams, D. A. 2001, MNRAS, 327, 1165

Furuya, R. S., Kitamura, Y., Wootten, A., Claussen, M. J., \& Kawabe, R. 2003, ApJS, 144, 71

Gensheimer, P. D., Mauersberger, R., \& Wilson, T. L. 1996, A\&A, 314, 281

Giannini, T., Nisini, B., \& Lorenzetti, D. 2001, ApJ, 555, 40

Goldsmith, P. F., \& Langer, W. D. 1978, ApJ, 222, 881

Green, S., Maluendes, S., \& McLean, A. D. 1993, ApJS, 85, 181

Grosjean, A., Dubernet, M.-L., \& Ceccarelli, C. 2003, A\&A, 408, 1197

Harwit, M., Neufeld, D. A., Melnick, G. J., \& Kaufman, M. J. 1998, ApJ, 497, L105

Helmich, F. P., van Dishoeck, E. F., Black, J. H., et al. 1996, A\&A, 315, L173

Hogerheijde, M. R., \& van der Tak, F. F. S. 2000, A\&A, 362, 697

Ivezić, Z., \& Elitzur, M. 1997, MNRAS, 287, 799

Jacq, T., Henkel, C., Walmsley, C. M., Jewell, P. R., \& Baudry, A. 1988, A\&A, 199, L5

Jacq, T., Walmsley, C. M., Henkel, C., et al. 1990, A\&A, 228, 447

Jørgensen, J. K. 2004, A\&A, 424, 589

Jørgensen, J. K., Schöier, F. L., \& van Dishoeck, E. F. 2002, A\&A, 389, 908 Jørgensen, J. K., Schöier, F. L., \& van Dishoeck, E. F. 2004, A\&A, 416, 603 Jørgensen, J. K., Schöier, F. L., \& van Dishoeck, E. F. 2005, A\&A, 437, 501 Jørgensen, J. K., Johnstone, D., van Dishoeck, E. F., \& Doty, S. D. 2006, A\&A, 449,609

Jørgensen, J. K., Bourke, T. L., Myers, P. C., et al. 2007, ApJ, 659, 479

Kaufman, M. J., \& Neufeld, D. A. 1996, ApJ, 456, 611

Lada, C. J. 1987, in Star Forming Regions, ed. M. Peimbert, J. Jugaku, \&

P. W. J. L. Brand (Dordrecht: Reidel), IAU Symp., 115, 1

Liseau, R., Ceccarelli, C., Larsson, B., et al. 1996, A\&A, 315, L181 
Maret, S., Ceccarelli, C., Caux, E., Tielens, A. G. G. M., \& Castets, A. 2002, A\&A, 395, 573

Maret, S., Ceccarelli, C., Caux, E., et al. 2004, A\&A, 416, 577

Motte, F., \& André, P. 2001, A\&A, 365, 440

Myers, P. C., Evans, II, N. J., \& Ohashi, N. 2000, Protostars and Planets IV, 217

Neufeld, D. A., \& Kaufman, M. J. 1993, ApJ, 418, 263

Neufeld, D. A., Snell, R. L., Ashby, M. L. N., et al. 2000, ApJ, 539, L107

Nisini, B., Benedettini, M., Giannini, T., et al. 1999, A\&A, 350, 529

Nisini, B., Giannini, T., \& Lorenzetti, D. 2002, ApJ, 574, 246

Ossenkopf, V., \& Henning, T. 1994, A\&A, 291, 943

Parise, B., Caux, E., Castets, A., et al. 2005, A\&A, 431, 547

Park, G., \& Choi, M. 2007, ApJ, 664, L99

Phillips, T. R., Maluendes, S., \& Green, S. 1996, ApJS, 107, 467

Poelman, D. R., \& Spaans, M. 2005, A\&A, 440, 559

Poelman, D. R., \& van der Tak, F. F. S. 2007, A\&A, 475, 949

Poglitsch, A., Waelkens, C., \& Geis, N. 2001, in The Promise of the Herschel Space Observatory, ed. G. L. Pilbratt, J. Cernicharo, A. M. Heras, T. Prusti, \& R. Harris, ESA SP-460, 29

Pontoppidan, K. M., van Dishoeck, E. F., \& Dartois, E. 2004, A\&A, 426, 925

Ristorcelli, I., Falgarone, E., Schöier, F., et al. 2005, in IAU Symp. 184, ed. D. C. Lis, G. A. Blake, \& E. Herbst

Schöier, F. L., Jørgensen, J. K., van Dishoeck, E. F., \& Blake, G. A. 2002, A\&A, 390,1001

Schöier, F. L., Jørgensen, J. K., van Dishoeck, E. F., \& Blake, G. A. 2004, A\&A, 418,185

Schöier, F. L., van der Tak, F. F. S., van Dishoeck, E. F., \& Black, J. H. 2005, A\&A, 432, 369

Shirley, Y. L., Evans, N. J., Rawlings, J. M. C., \& Gregersen, E. M. 2000, ApJS, 131,249
Shu, F. H. 1977, ApJ, 214, 488

Smith, R. G., Sellgren, K., \& Tokunaga, A. T. 1989, ApJ, 344, 413

Snell, R. L., Howe, J. E., Ashby, M. L. N., et al. 2000, ApJ, 539, L101

Stark, R., Sandell, G., Beck, S. C., et al. 2004, ApJ, 608, 341

Turner, B. E., Fourikis, N., Morris, M., Palmer, P., \& Zuckerman, B. 1975, ApJ, 198, L125

van der Tak, F. F. S., van Dishoeck, E. F., Evans, N. J., \& Blake, G. A. 2000, ApJ, 537, 283

van der Tak, F., Neufeld, D., Yates, J., et al. 2005, in ESA SP-577, ed. A. Wilson, 431

van der Tak, F. F. S., Walmsley, C. M., Herpin, F., \& Ceccarelli, C. 2006, A\&A, 447, 1011

van Dishoeck, E. F. 2003, in SFChem 2002: Chemistry as a Diagnostic of Star Formation, Proc. Conf. held August 21-23, 2002 at University of Waterloo, Ontario, Canada, ed. C. L. Curry, \& M. Fich (Ottawa, Canada: NRC Press), 201

van Dishoeck, E. F., \& Blake, G. A. 1998, ARA\&A, 36, 317

van Dishoeck, E. F., Blake, G. A., Draine, B. T., \& Lunine, J. I. 1993, in Protostars and Planets III, ed. E. H. Levy, \& J. I. Lunine, 163

van Zadelhoff, G.-J., Dullemond, C. P., van der Tak, F. F. S., et al. 2002, A\&A, 395,373

Ward-Thompson, D., \& Buckley, H. D. 2001, MNRAS, 327, 955

Watson, D. M., Bohac, C. J., Hull, C., et al. 2007, Nature, 448, 1026

Whittet, D. C. B., Bode, M. F., Baines, D. W. T., Longmore, A. J., \& Evans, A. 1983, Nature, 303, 218

Wilson, T. L., \& Rood, R. 1994, ARA\&A, 32, 191

Young, C. H., \& Evans, II, N. J. 2005, ApJ, 627, 293 
T. A. van Kempen et al.: Modeling water emission from low-mass protostellar envelopes, Online Material p 1
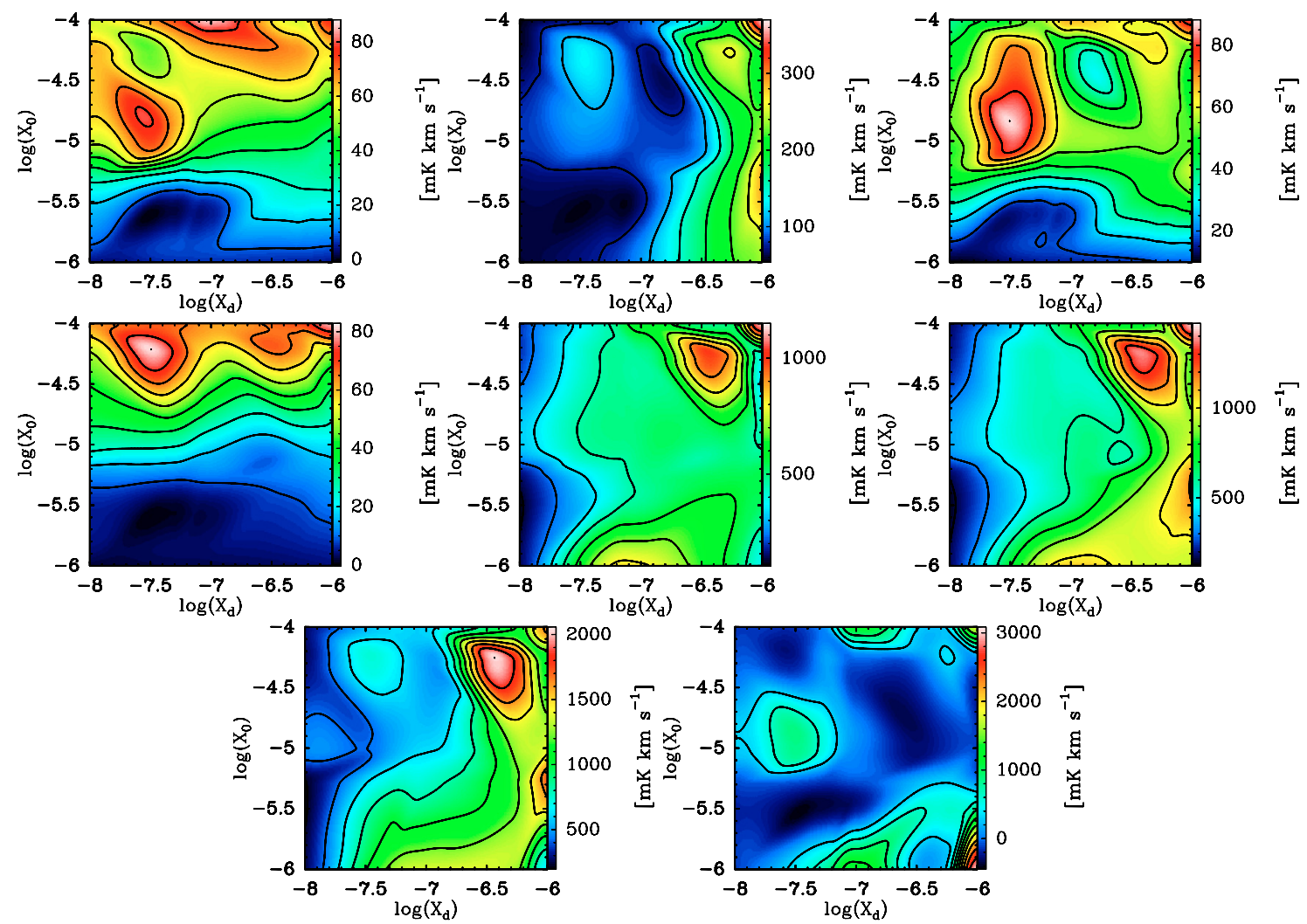

Fig. 11. Contour plots of the integrated intensity of the $\mathrm{L} 483$ models as a function of inner $\left(X_{0}\right)$ and outer $\left(X_{\mathrm{d}}\right.$ for all para- $\mathrm{H}_{2} \mathrm{O}$ lines. Upper row (from left to right): $5_{24}-4_{31}, 4_{22}-4_{13}$ and $4_{22}-3_{31}$. Middle row: $3_{31}-4_{04}, 2_{20}-2_{11}$ and $2_{11}-2_{02}$. Lower row: $2_{02}-1_{11}$ and $1_{11}-0_{00}$.
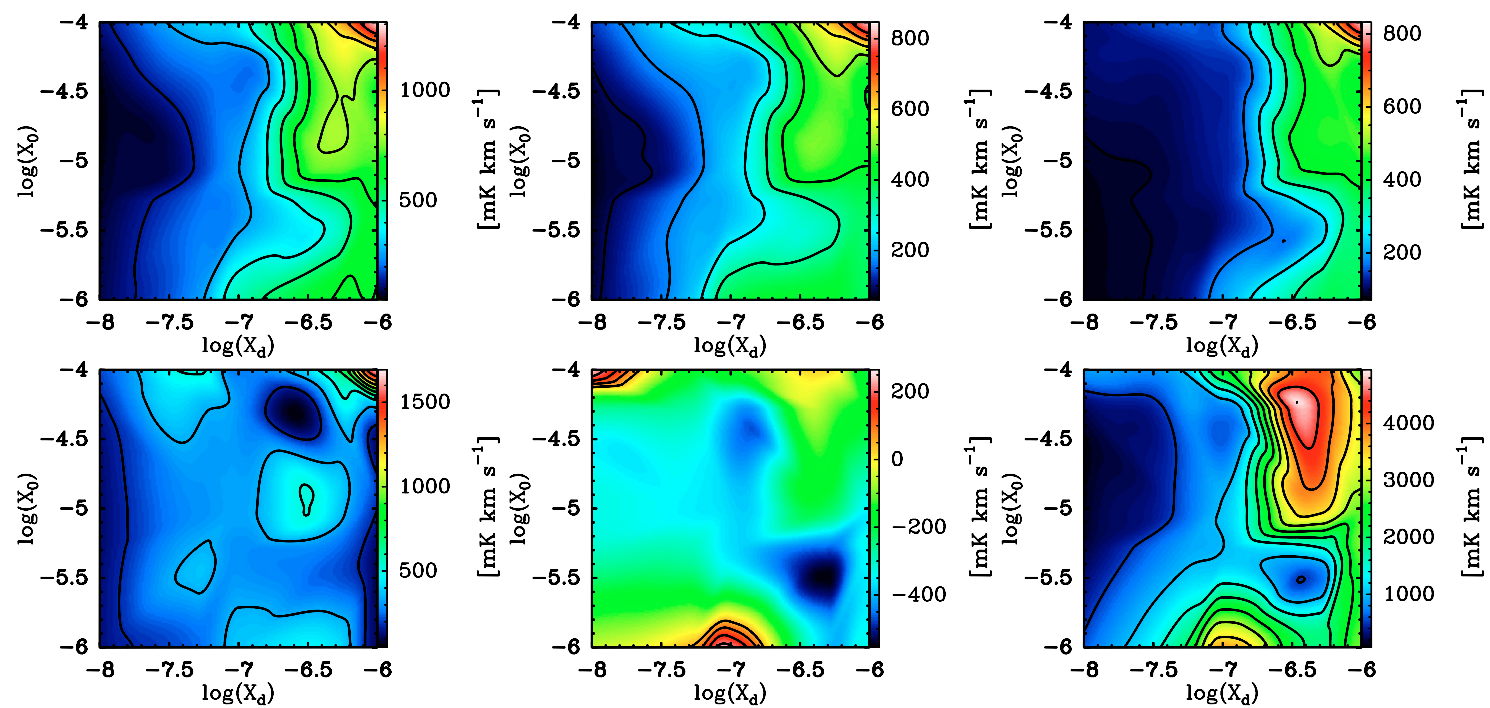

Fig. 12. Contour plots of the integrated intensity of the $\mathrm{L} 483$ models as a function of inner $\left(X_{0}\right)$ and outer $\left(X_{\mathrm{d}}\right.$ for all ortho- $\mathrm{H}_{2} \mathrm{O}$ lines. Upper row (from left to right): $3_{21}-3_{12}, 3_{12}-3_{03}$ and $3_{12}-2_{21}$. Lower row: $2_{21}-2_{12}, 2_{12}-1_{01}$ and $1_{10}-1_{01}$. 
T. A. van Kempen et al.: Modeling water emission from low-mass protostellar envelopes, Online Material p 2

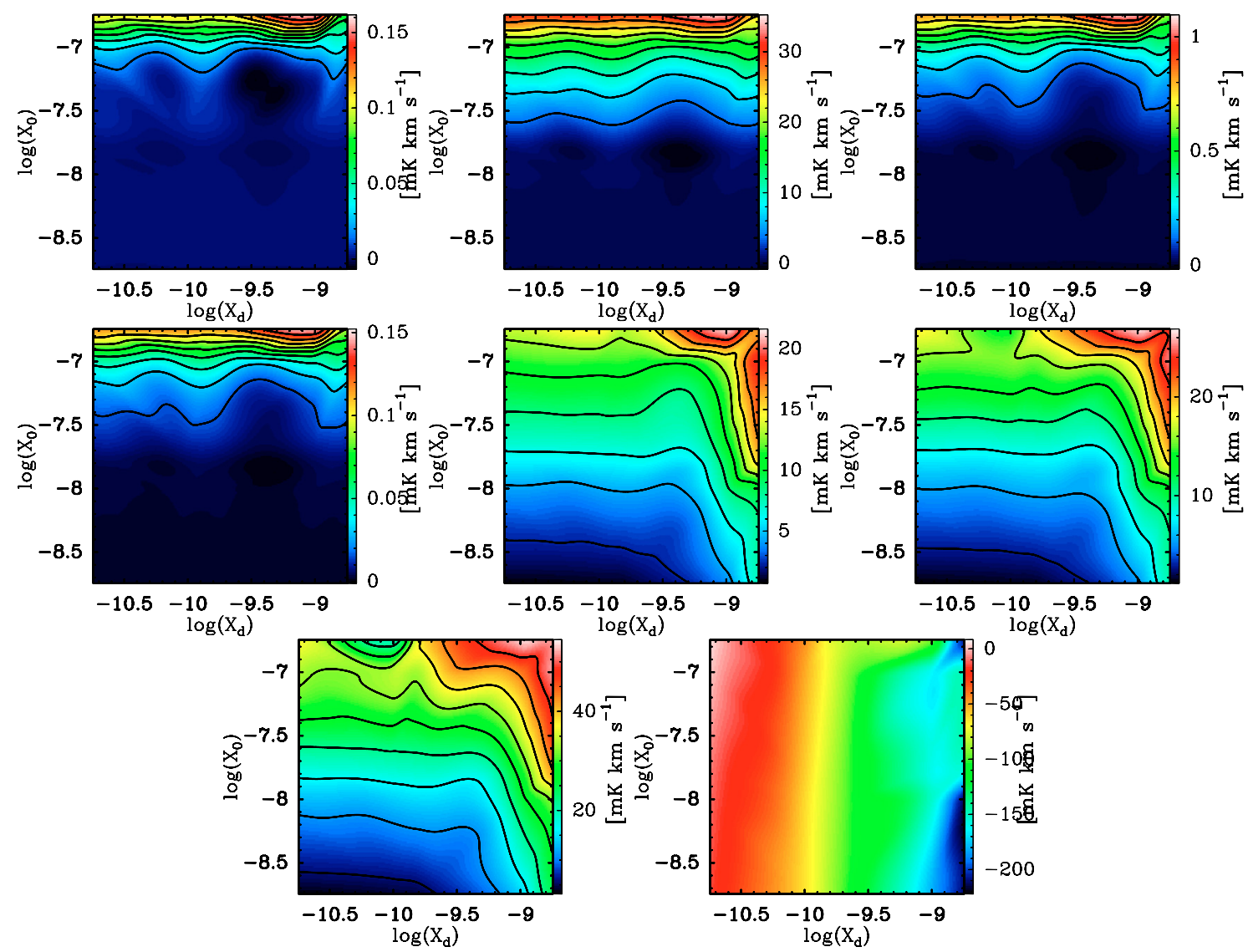

Fig. 13. Contour plots of the integrated intensity of the $\mathrm{L} 483$ models as a function of inner $\left(X_{0}\right)$ and outer $\left(X_{\mathrm{d}}\right.$ for all para- $\mathrm{H}_{2}^{18} \mathrm{O}$ lines. Upper row (from left to right): $5_{24}-4_{31}, 4_{22}-4_{13}$ and $4_{22}-3_{31}$. Middle row: $3_{31}-4_{04}, 2_{20}-2_{11}$ and $2_{11}-2_{02}$. Lower row: $2_{02}-1_{11}$ and $1_{11}-0_{00}$.
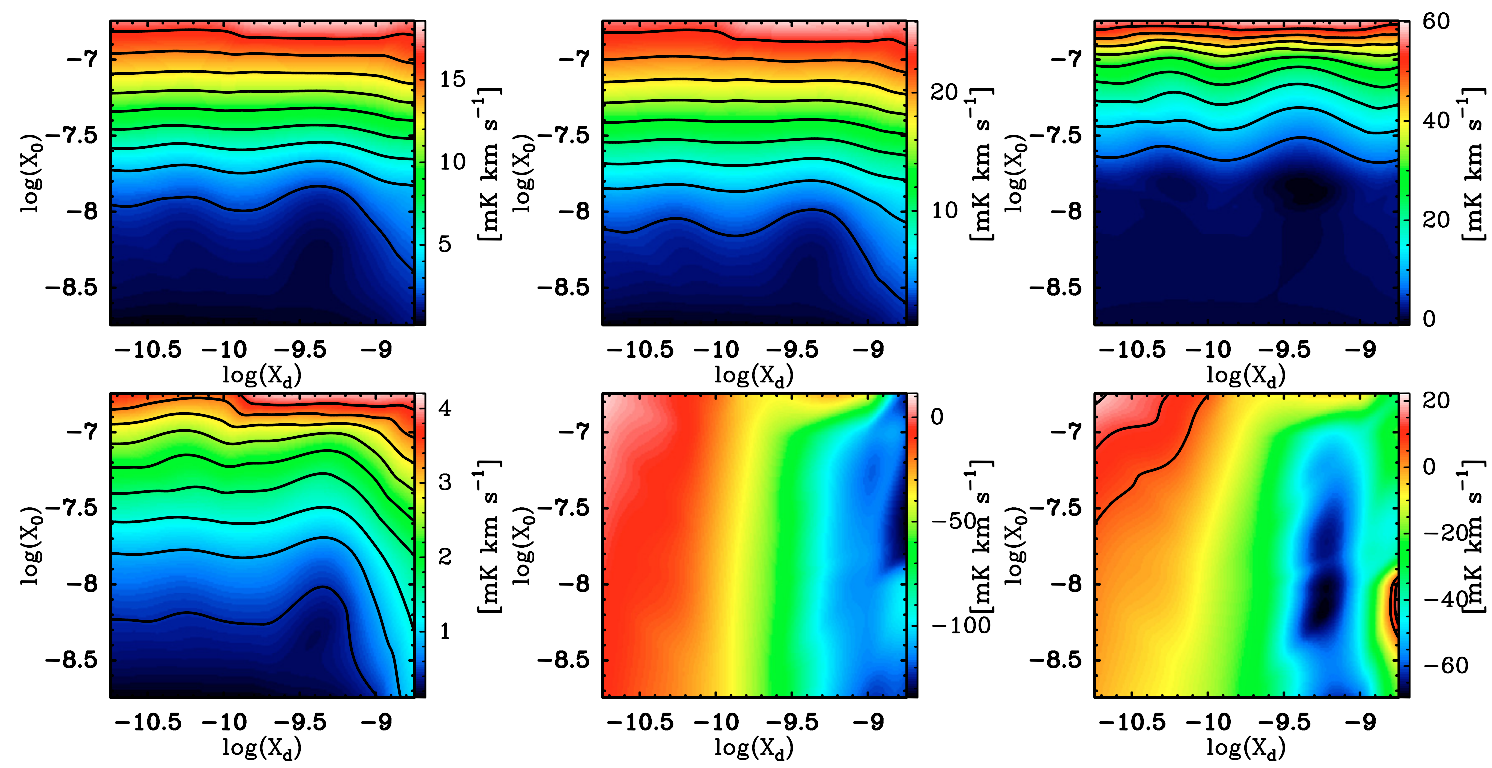

Fig. 14. Contour plots of the integrated intensity of the $\mathrm{L} 483$ models as a function of inner $\left(X_{0}\right)$ and outer $\left(X_{\mathrm{d}}\right.$ for all ortho- $\mathrm{H}_{2}^{18} \mathrm{O}$ lines. Upper row (from left to right): $3_{21}-3_{12}, 3_{12}-3_{03}$ and $3_{12}-2_{21}$. Lower row: $2_{21}-2_{12}, 2_{12}-1_{01}$ and $1_{10}-1_{01}$. 
T. A. van Kempen et al.: Modeling water emission from low-mass protostellar envelopes, Online Material p 3

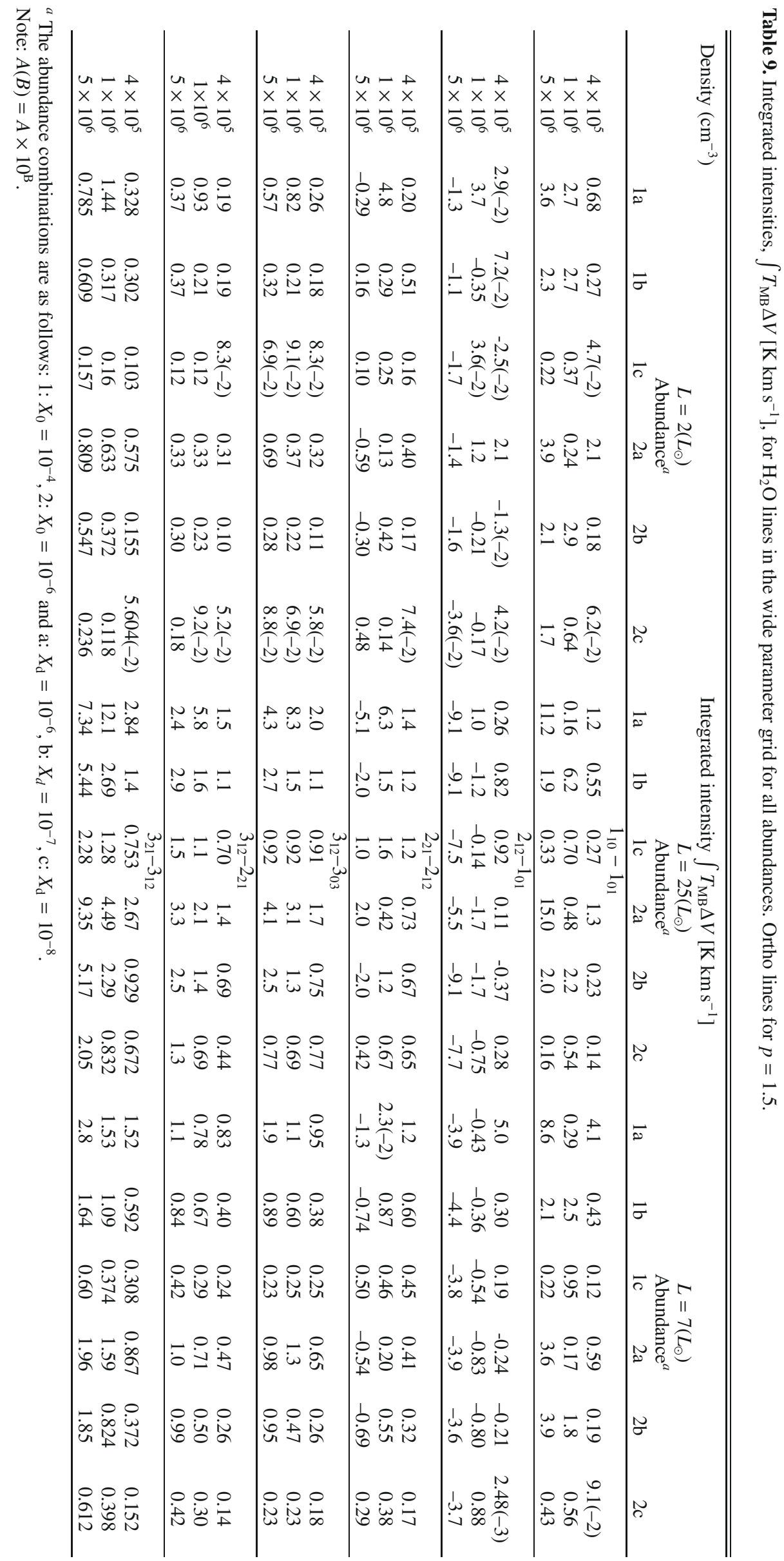


T. A. van Kempen et al.: Modeling water emission from low-mass protostellar envelopes, Online Material $p 4$

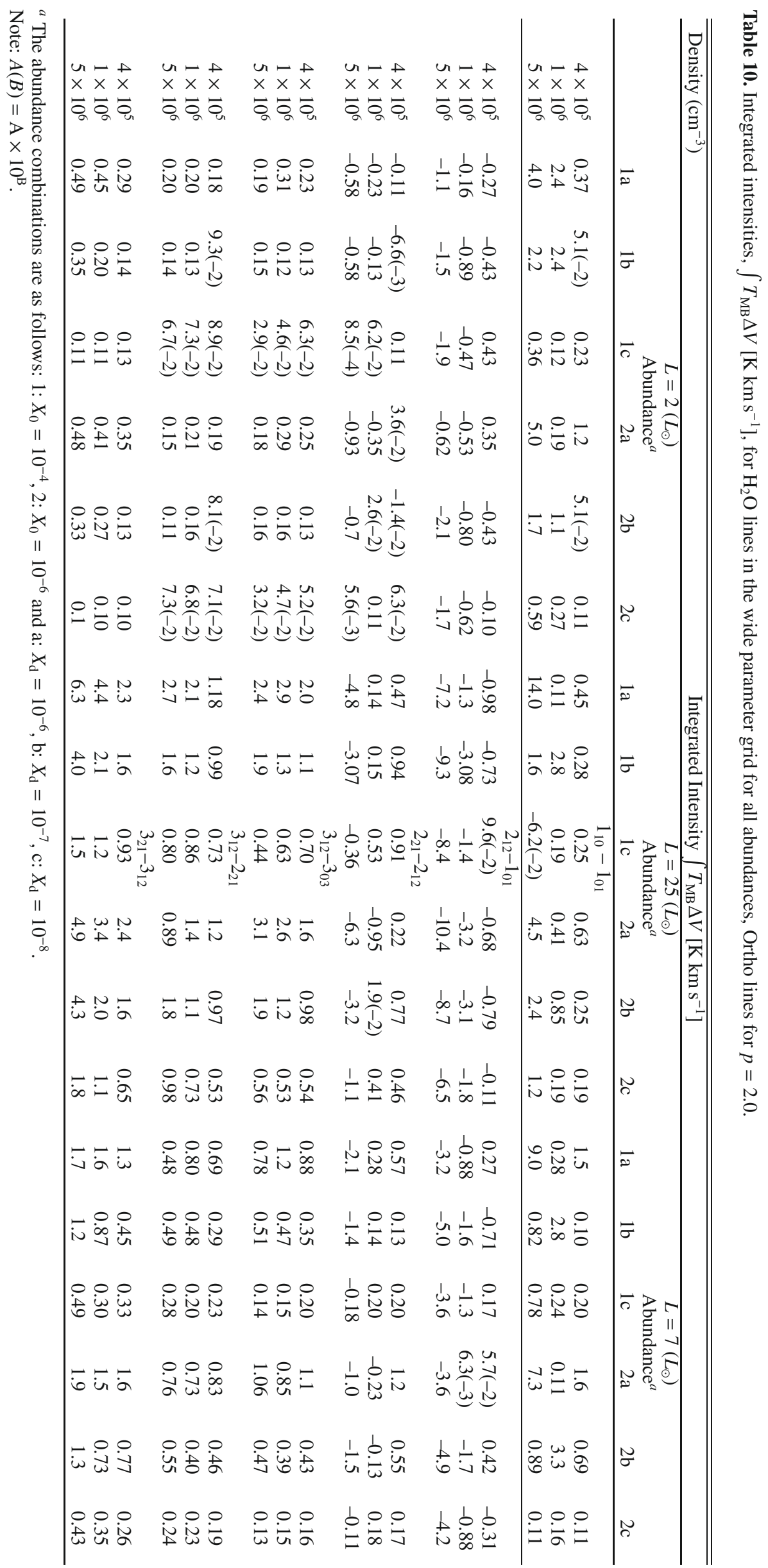


T. A. van Kempen et al.: Modeling water emission from low-mass protostellar envelopes, Online Material p 5

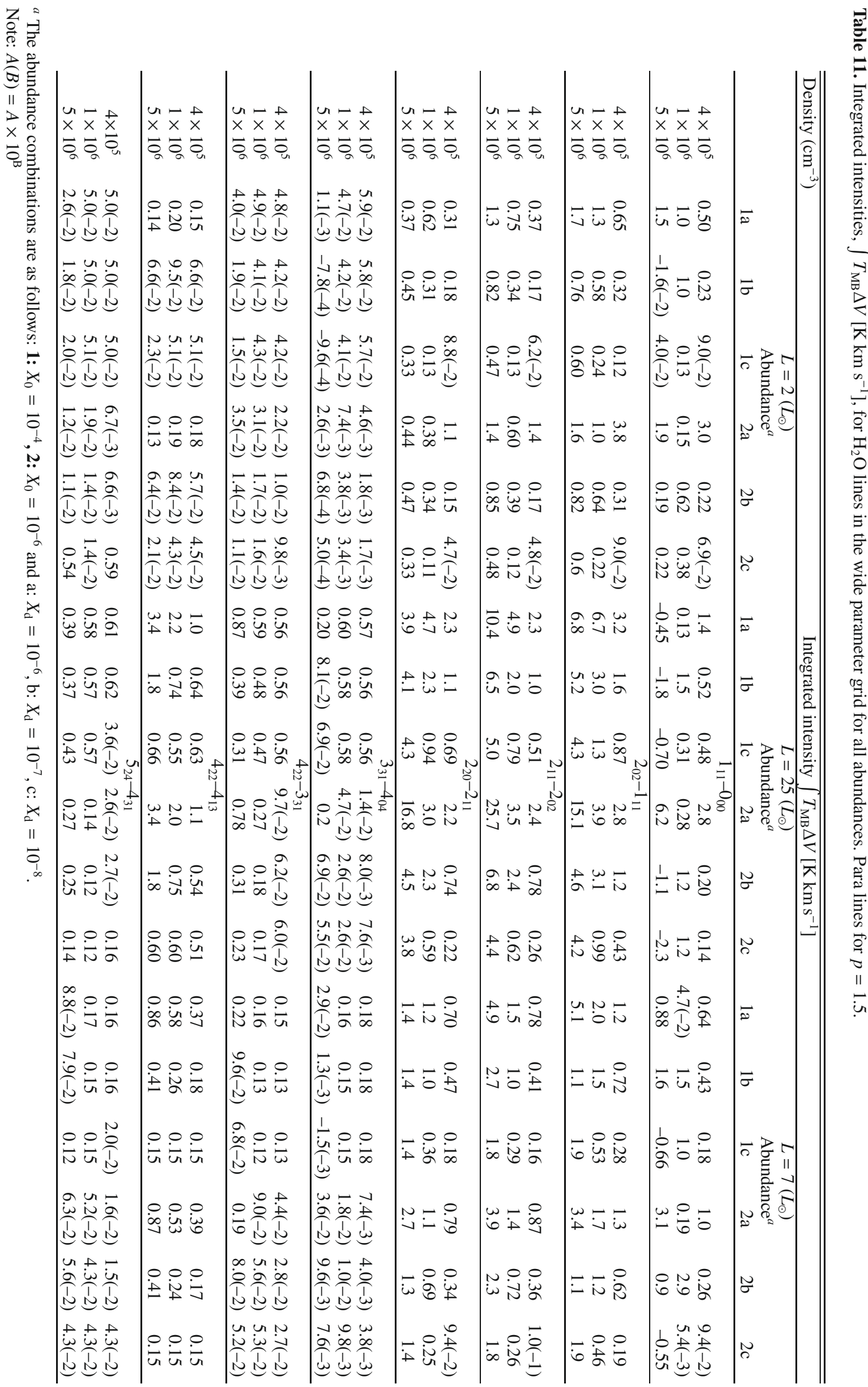


T. A. van Kempen et al.: Modeling water emission from low-mass protostellar envelopes, Online Material p 6

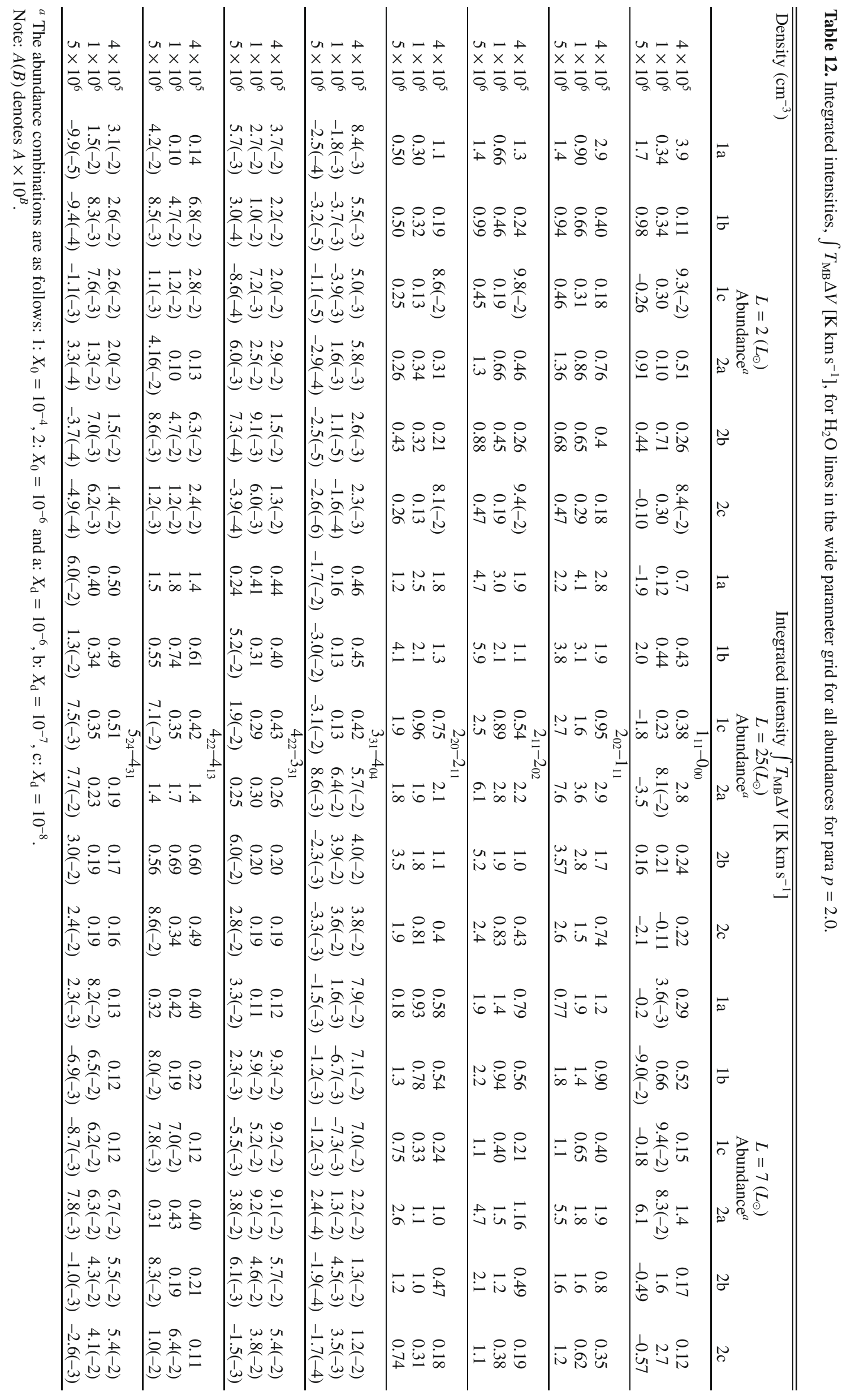

